\title{
Suspension in Convective Layers and Style of Differentiation of a Terrestrial Magma Ocean
}

\author{
Viatcheslav S. Solomatov and David J. Stevenson
}

Division of Geological and Planetary Sciences, California Institute of Technology, Pasadena

\begin{abstract}
Recent physical theories for the formation of the Earth suggest that about 4.5 b.y. ago the mantle of the Earth was partially or completely molten. Fractional crystallization of this hypothetical magma ocean would result in a strong chemical stratification of the Earth's mantle. Such a scenario is controversial from the geochemical point of view. However, it has been noted that the simple scenario of fractional crystallization could be avoidable in a convective magma ocean if crystals remain suspended. In this paper, the problem of suspension is developed with the help of an energetic approach: convection must do some work against gravitational settling. We distinguish three regimes of convective suspensions. Absolute or complete sedimentation accurs when the energy dissipation due to the settling exceeds the heat loss from the convective layer. This is possible only in large-scale systems like magma oceans and implies that cooling can proceed only together with sedimentation, crystallization, and a decrease in the liquidus temperature at a constant pressure. A regime of partial differentiation occurs when the energy dissipation due to the settling is less than the total heat loss but larger than the power which can be spent by convection on the crystal reentrainment process. The differentiation is not complete, and a competition between the rate of cooling, the rate of sedimentation, and the rate of turbulent diffusion determines the degree of differentiation. The third regime is an absolute suspension which could be sustained for an indefinitely long time. In this case, sedimentation starts only when the crystal fraction reaches the maximum packing value: when the viscosity of the magma rapidly increases. The power which can be spent by convection on reentrainment is equal to $\epsilon \alpha g d / c_{p}$ of the total energy supply to the convective layer, where $\epsilon<1$ is an efficiency factor. This factor is probably about 0.01 and has been estimated from one experiment on convective suspensions and with the help of an analogy with remixing in chemically layered convective layers; we find that both cases are controlled by the energetics of convection. The crystal radius is one of the most crucial and uncertain parameters. If it exceeds about $10^{-2}-1 \mathrm{~cm}$ during crystallization of deep layers (>15 GPa) or $10^{-3}-10^{-1} \mathrm{~cm}$ during crystallization of shallow layers, the first regime ("fractional crystallization") is unavoidable. The estimates depend on various poorly constrained parameters and processes, such as heat flux, viscosity, thermodynamical disequilibrium and highly variable viscosity convection. For absolute suspension the crystal size must be at least $\epsilon^{1 / 2}$ times less, or $10^{-3}-10^{-1} \mathrm{~cm}$ and $10^{-4}-10^{-2} \mathrm{~cm}$, respectively, if $\epsilon \sim 0.01$. The partial differentiation occurs in a narrow (one decade) range between these two regimes. The radius of about $1 \mathrm{~cm}$ must be considered as an absolute upper bound above which fractional differentiation is guaranteed. These estimates for the critical crystal size are orders of magnitude lower than suggested previously, and thus the problem of crystal sizes becomes a central one for magma oceans. A necessary condition for reentrainment is the existence of local mechanisms. The absence of such mechanisms to reentrain the particles from the bottom would mean that an absolute suspension is impossible even if the energetics allows it. Turbulence is considered as a possible important factor. A simple model of convection predicts a strong turbulence, provided the viscosity is less than $10^{9}-10^{10}$ $\mathrm{P}$. Rotation reduces this critical viscosity to $10^{5}-10^{8} \mathrm{P}$ but this is still sufficiently large and is reached only near the maximum packing crystal fraction. Power law or Bingham rheology of partial melts can exclude any turbulence already at $20-30 \%$ of crystal fraction. We also show that the energetic criterion for the absolute suspension with $\epsilon \sim 1$ coincides with the condition that the particle concentration gradient suppresses the turbulence.
\end{abstract}

\section{INTRODUCTION}

Various arguments suggest that the Earth was once mostly or entirely molten. The first argument results from the recent accretion theories [Safronov, 1978; Wetherill, $1985,1990]$. The population of planetesimals from which the Earth and the planets formed was not uniform but contained bodies of different sizes. The largest bodies could be about the size of Mars. Collisions between the Earth and the planetesimals of this size unavoidably caused global melting of the Earth and even partial vaporization [Cameron and Benz, 1989; Benz and Cameron, 1990; Melosh, 1990]. A Mars-size impactor is believed to be needed in the impact

Copyright 1993 by the American Geophysical Union.

Paper number 92JE02948.

0148-0227/93/92JE-02948\$05.00 theory of the lunar origin [Stevenson, 1987; Newsom and Taylor, 1989; Benz et al., 1986, 1987, 1989; Cameron and Benz, 1991].

Another argument is that a dense, steam atmosphere maintained a very high surface temperature during accretion, allowing an upper molten layer of the mantle [Abe and Matsui, 1986; Matsui and Abe, 1986; Zahnle et al., 1988; Kasting, 1988].

The temperature increase due to gravitational differentiation of iron [Flasar and Birch, 1973; Verhoogen, 1980] simultaneously with adiabatic compression and heating by short-living radiogenic isotopes [e.g., Safronov, 1978] can also produce melting of some part of the mantle. However, these effects become less important if the giant impacts took place.

While the existence of a magma ocean seems to be unavoidable from the point of view of the formation theories, it is controversial from the geochemical point of view [see 
reviews by Ringwood, 1990; Taylor and Norman, 1992; Solomatov and Stevenson, this issue (a)]. Existing geochemical models assume a simplified physical scheme of crystallization of magma oceans that is essentially a fractional crystallization with strong differentiation. However, it is unclear whether or not this style of differentiation can take place in magma oceans. As has been pointed out by Tonks and Melosh [1990], convection in magma oceans can play an important role in preventing the differentiation.

A central problem of differentiation of magma oceans is suspension in convective layers. Suspension of particles in a convective layer has been studied with the help of several different approaches. One approach is based on a phenomenological equation for the balance between the downward settling flux of particles due to the gravity and the upward flux due to the convective diffusivity [Barlett, 1969; Huppert and Sparks, 1980]. This approach has been applied to magma oceans by $A b e[1991,1992]$. Although it can be used to find solutions for the particle distribution, it does not establish criteria for suspension; different assumptions about the boundary conditions can allow or forbid suspension.

In the other approach, the regions with closed particle trajectories in a steady convective flow are considered as the regions of suspension [Marsh and Maxey, 1985; Weinstein et al., 1988; Rudman, 1992]. The problem is that these closed trajectories are stable only for these given flows. Perturbations (in chaotic cases) can move particles from these trajectories to those that bring them to the lower boundary of the convective layer. If there is no reentrainment mechanism (as in the models considered), every particle has a nonzero probability to reach the bottom and remain there. Eventually, all the particles must settle out.

Laboratory experiments by Martin and Nokes [1988, 1989] show that even in the case when the settling velocity is much smaller than the convective velocity, all the particles may eventually settle. They argue that because the convective velocity at the lower boundary vanishes, the particles cannot reenter the internal region and remain at this boundary. An important exception will be discussed later.

Tonks and Melosh [1990] suggest that the turbulence is an essential factor and suspension occurs if the frictional velocity in the turbulent boundary layers is larger than the settling velocity of the particles. This analysis is possibly applicable to a shear turbulence, but the physics of the suspension in convective layers is quite different as we discuss in the present paper.

Usually the influence of the particles on the convective flow was ignored. However, Koyaguchi et al. [1990] find that already above some small (a fraction of percent) critical solid fraction this influence is so big that the suspended particles suppress convection and create a layered convective system. These results were confirmed both experimentally and theoretically. A similar effect has been observed in a numerical study by Rudman [1992]. Thus, the effect of suspension on convection could be crucial.

The present study is organized in the following way: (1) we develop a theory of convective suspensions and show that the suspension in a convective layer is controlled by the energetics of convection. (2) The theory is compared with the experiments by Martin and Nokes $[1988,1989]$ where there was one reentrainment case. (3) The same energetic approach is applied to the problem of chemical erosion in convective layers for which there are more data [Olson, 1984].
(4) The sedimentation regimes studied by Koyaguchi et al. [1990] and Rudman [1992] are discussed from the perspective of our theory. (5) The theory is applied to a magma ocean, and conditions for different regimes of suspension and sedimentation are suggested. (6) Turbulence as one of the local mechanisms responsible for reentrainment is studied in the case of convection with rotation and non-Newtonian rheology. (7) The stabilizing effect of particle concentration gradient on turbulent convection is studied in the last section.

\section{Suspension of Particles in a Convective Layer}

The relative motion between the liquid and the particles suspended in a convective layer always takes place independently of whether or not particles sediment at the bottom. If only the gravity effects are taken into account, then we have a permanent gravitational settling. To sustain suspension, convection must do some work. On the other hand, this power is limited. Thus, the necessary criterion is that the work per unit time which can be done by convection must be larger than the gravitational work per unit time required to suspend the particles.

The work $E_{c}$ per unit time done by highly developed convection is equal to the viscous dissipation and is expressed as [Golitsyn, 1978; Solomatov, 1993]:

$$
\begin{gathered}
E_{c}=\int_{V} \tau_{i j} \frac{\partial u_{i}}{\partial x_{j}} d V=-\int_{V} \alpha g \rho\left(T-T_{0}\right) u_{z} d V= \\
D\left(A F_{B}+\frac{1}{d} \int_{0}^{d} \int_{0}^{z^{\prime}} \int_{A} Q d A d z d z^{\prime}\right),
\end{gathered}
$$

where

$$
D=\frac{\alpha g d}{c_{p}}
$$

is the ratio of the thickness of the layer $d$ to the adiabatic temperature scale $c_{p} / \alpha g$ and it is also known as the dissipation parameter, $\tau_{i j}$ is the deviatoric stress tensor, $u_{i}$ is the velocity vector, $u_{z}$ is the vertical component of the velocity vector, $d$ is the thickness of the convective layer, the thermodynamical parameters must be calculated as the effective ones for the multiphase medium [Solomatov and Stevenson, this volume $(a)], F_{B}$ is the bottom heat flux, $A$ is the horizontal area of the layer, $z$ and $z^{\prime}$ denote the vertical coordinate, $Q$ is the internal heating per unit volume, $g$ is the acceleration due to the gravity, $\rho$ is the averaged density of the system, $c_{p}$ is the thermal capacity per unit mass, $\alpha$ is the thermal expansion, and particle settling is ignored for the moment. Note that a triple integration of the equations of thermal convection is necessary to obtain the correct formula [Golitsyn, 1978].

This integral is valid for both laminar and turbulent convection and was found for a plane horizontal layer in the case of steady heating for $D \ll 1$ (shallow layers). It can be shown that for the unsteady heating case the term $-\rho c_{p} \partial T / \partial t$ must be added to $Q$, which can be interpreted as an effective heat production due to the cooling. (We note, however, that in a strict derivation there is also another term $c_{p} u_{i} \partial T / \partial x_{\imath}$, which gives zero contribution only if $c_{p}=$ const, or only if $\partial c_{p} / \partial x=\partial c_{p} / \partial y=0$.)

In the presence of the particles the equations are more complicated. The particle motion in a turbulent flow is described with the help of Tsen's equation [e.g., Soo, 1967]. 
An approximation to Tsen's equation in which only the terminal velocity due to their buoyancy is taken into account is possibly a sufficient one [see discussion by Marsh and Maxey, 1985]. In the first approximation we can take into account only this physically important effect: the relative motion between the particles and the liquid due to the gravitational settling. The work per unit time done by convection is now equal to

$$
\begin{gathered}
E_{c}=\frac{\alpha g d}{c_{p}}\left(A F_{B}+V Q^{\prime}\right) \\
Q^{\prime}=\frac{1}{d^{2} A} \int_{0}^{d} \int_{0}^{z^{\prime}} \int_{A}\left(Q-\rho c_{p} \frac{\partial T}{\partial t}+e_{p}\right) d A d z d z^{\prime}
\end{gathered}
$$

where $A$ is the surface area, and $e_{p}$ is the energy dissipation due to the particle settling per unit volume.

In the case of highly developed convection the convective layer is almost adiabatic (or isothermal if $D \ll 1$ ) except for negligibly thin thermal boundary layers. Thus the term with $\rho c_{p} \partial T / \partial t$ can be approximated by an averaged temperature drop rate. Assuming also a uniform initial distribution of particles, we find

$$
E_{c}=\frac{\alpha g d}{c_{p}} A \frac{\left(F_{B}+F\right)}{2}
$$

where $F$ is the heat flux at the top of the convective layer:

$$
F A=F_{B} A+M\left(Q-\rho c_{p} \frac{d \bar{T}}{d t}\right)+E_{p},
$$

$\bar{T}$ is the averaged temperature of the layer, and $M$ is the mass of the magma ocean.

The integral in ( 3 ) is estimated as

$$
E_{c} \approx \frac{\alpha g d}{c_{p}} A F=D A F, D \ll 1 .
$$

In the case of the deep multiphase magma ocean the dissipation parameter $D$ is of the order of 1 (see below) and the above equations become less accurate. In this case, $E_{c}$ can still be estimated as

$$
E_{c} \approx A F, \quad D \sim 1 .
$$

The gravitational work per unit time $E_{p}$ required to suspend the crystals is equal to

$$
E_{p}=u_{p} g M_{p} \frac{\Delta \rho}{\rho}=u_{p} g \phi M \frac{\Delta \rho}{\rho}
$$

where $u_{p}$ is the settling velocity of the particles, $M_{p}=\phi M$ is the mass of the particles, $\phi$ is the mass fraction of the particles, $\Delta \rho$ is the density difference between the particles and liquid, and the ratio $\Delta \rho / \rho$ is supposed to be small.

The necessary criterion for suspension is

$$
E_{p}<E_{c} \text {. }
$$

When the Reynolds number for the particle settling and the crystal fraction are both small, then Stokes' formula [Landau and Lifshitz, 1989] is valid:

$$
u_{p}=\frac{2 g \Delta \rho r^{2}}{9 \eta_{0}},
$$

where $r$ is the particle radius, and $\eta_{0}$ is the viscosity of the liquid.

The criterion ( 10 ) written for the particle radius becomes

$$
r<\left(\frac{9 \alpha \eta_{0} F}{2 \Delta \rho^{2} g \phi c_{p}}\right)^{1 / 2}, \phi \ll 1, \quad D<1,
$$

where the sphericity of the mantle was ignored and so $M \approx$ $\rho d A$.

If the crystal fraction is larger than about 0.1 , then the Ergun-Orning formula must be used [Soo, 1967]. It can be simplified to

$$
u_{p}=\frac{4 g \Delta \rho r^{2}(1-\phi)^{2}}{150 \eta_{0} \phi}
$$

Note that in the case of shallow layers this equation can be applied up to $\phi \approx \phi_{m}$, where $\phi_{m}$ is the maximum packing solid fraction. In the case of deep layers (much larger than compaction length [McKenzie, 1984]) this formula is possibly valid until $\phi \sim 1$. In this case it describes the percolation law for the flow in porous media.

In terms of the ratio of the settling velocity of the particles $u_{p}$ to the convective velocity (Rouse number),

$$
S=\frac{u_{p}}{u_{c}}
$$

the above criteria are rewritten as follows. For laminar convection in the case $\eta \approx$ const ( $\eta$ is the viscosity of the crystal-melt system) the theory and experiments give [Golitsyn, 1978; Turcotte and Schubert, 1982]

$$
u_{c} \sim\left(\frac{\alpha g d}{c_{p}} \frac{d F}{\eta}\right)^{1 / 2} .
$$

Ignoring a numerical factor, we can write the criteria for suspension as

$$
S<\frac{r}{d} \frac{1}{\phi^{1 / 2}}, \phi \ll 1
$$

For the turbulent convection, using (43), we obtain

$$
S<\frac{r}{d} \frac{R e^{1 / 2}}{\phi^{1 / 2}}, \phi \ll 1,
$$

The criterion derived gives only the upper bound for the suspension in convective layers because we do not know which fraction of the total power is spent on sustaining the suspension. In the following sections we will show that this fraction is possibly only about $1 \%$. We define an efficiency factor $\epsilon$ which is equal to the fraction of the maximum available power of the convection that is spent on the gravitational work against sedimentation. Thus, the equilibrium state in which the sedimentation is exactly compensated by the convective "resuspension" is described by the equation

$$
E_{p}=\epsilon E_{c} \text {. }
$$

Thus while the usual efficiency of convection (the ratio of the total work per unit time to the total energy supply) is proportional to the parameter $D=\alpha g d / c_{p}$ (see ( 5 ) and also works by Hewitt et al. [1975] and Golitsyn [1978]), the efficiency of convection for the suspension of particles is proportional to $\epsilon D$.

Note that in the limit $D \sim 1$, the upper bound for the suspension is equivalent to the requirement that the heat production due to the particle settling must be simply smaller than the heat flux from the layer. This limit is relevant to magma oceans.

\section{ANalogy Between Erosion and Suspension IN CONVECTIVE LAYERS}

Olson [1984] studied the problem of erosion in a twolayered convective system. Two layers of equal thickness 
had a stable density contrast due to different chemical composition and were heated from below and cooled from above. The mixing between these two layers due to a slow erosion at the interface was studied. The experimental results can be rewritten in the following form:

$$
\frac{1}{\Delta \rho} \frac{d \Delta \rho}{d t}=-0.05 \frac{\alpha F}{\Delta \rho d}
$$

where $\rho$ is a mean density of the layers, $\Delta \rho$ is the density contrast between the layers, $d$ is the total thickness of the two-layered system, and $F$ is the heat flux.

Below we show that this formula follows from the energetics of convection, as in the case of the suspension problem. The approach is similar to that of Olson [1984], but it is based on a more strict energetic equation [Golitsyn, 1978] used in the previous section. It is also worth noting that Sleep [1988] obtained a similar formula (if the coefficients are ignored) when considering the carrying ability of plumes.

The total gravitational energy of the system is equal to

$$
E_{G}=g A\left[\rho_{1} d_{1}\left(d_{2}+\frac{d_{1}}{2}\right)+\frac{\rho_{2} d_{2}^{2}}{2}\right],
$$

where index " 1 " refers to the upper layer and index " 2 " to the lower layer, $A$ is the surface area.

During the erosion the total thickness of the layer is constant and the total mass is also conserved. After some simple manipulations, we find an expression for $d E_{G} / d t$ for the small time intervals (when the changes are small):

$$
\frac{d E_{G}}{d t}=-\frac{1}{8} g A d^{2} \frac{d \Delta \rho}{d t} .
$$

Note that the energy increases with time. This is the result of the work done by convection. According to our theory, the work per unit time which can be done by convection is expressed as follows:

$$
E_{\mathrm{conv}}=\epsilon \frac{\alpha g d}{c_{p}} A F,
$$

which is applied to the total two-layered system.

From the equation

$$
E_{\mathrm{conv}}=\frac{d E_{G}}{d t}
$$

we immediately obtain that

$$
\frac{1}{\Delta \rho} \frac{d \Delta \rho}{d t}=-8 \epsilon \frac{\alpha F}{\Delta \rho d}
$$

which is exactly the equation ( 19) found by Olson [1984]. The efficiency factor $\epsilon$ is equal to

$$
\epsilon \approx 0.6 \% \text {. }
$$

Thus the energetic approach gives the right formula for the erosion problem, and the efficiency factor $\epsilon$ is constant.

\section{Comparison With Martin and NoKes [1988, 1989]}

In the experiments by Martin and Nokes [1988, 1989] there was no reentrainment except for one case. Below we consider two questions. Did a turbulence as a possible local mechanism of reentrainment [Tonks and Melosh, 1990] take place in their experiments? The second question is: can our theory explain the absence of reentrainment in most experiments and the observed reentrainment in one experiment? Table 1A of Martin and Nokes [1989] includes the experimental parameters, among which are the Rayleigh number $R a$, the Prandtl number $P r$, the Nusselt number $N u$, the Rouse number $S$, and the rms vertical component of the convective velocity $w_{\text {rms }}$. The ratio of the thetmal diffusivity $\kappa$ to the thickness of the layer $d$ is equal to $7.05 \times 10^{-5} \mathrm{~cm} \mathrm{~s}^{-1}$. The Reynolds number is calculated as

$$
R e=\frac{\rho w_{\mathrm{rms}} d}{\eta}=\frac{w_{\mathrm{rms}} d}{\kappa P r}
$$

and covers the range $R e=0.03-1650$ (see also Table 1 for a part of the experiments). Laminar and turbulent regimes are separated by the critical value of the Reynolds number about 30-40 [e.g., Kraichnan, 1962] ( and see also the following sections) and thus both regimes were studied. The reentrainment was observed at the lowest Reynolds number $R e=0.03$ and certainly took place in the laminar regime. This means that the turbulence was not the crucial factor in this particular case.

For a quantitative comparison with our theory, we consider only the case where the convection is driven by heating from below and cooling from above. This is the case where the reentrainment was observed.

We have to rearrange all the criteria in such a way that they would involve the known parameters. The critical solid fraction $\phi_{\mathrm{cr}}$ for an interface formation [Koyaguchi et al., 1990] (see the next section) is calculated as

$$
\phi_{\mathrm{cr}}=\frac{\rho \alpha \Delta T}{\Delta \rho}=\frac{2}{9}\left(\frac{r}{d}\right)^{2} \frac{\kappa}{d} \frac{R a}{w_{\mathrm{rms}} S},
$$

where $\alpha$ is the thermal expansion and $\Delta T$ is the temperature difference across the layer.

The equilibrium solid fraction $\phi_{\text {eq }}$ is expressed from (12) in terms of tabulated parameters as

$$
\phi_{\mathrm{eq}}=\epsilon \frac{9 \alpha \eta_{\mathrm{o}} F}{2 \Delta \rho^{2} g r^{2} c_{p}}=\frac{2}{9}\left(\frac{r}{d}\right)^{2}\left(\frac{\kappa}{d}\right)^{2} \frac{\epsilon R a N u}{w_{\mathrm{rmb}}^{2} S^{2}} .
$$

Unfortunately, the crystal radius and the density difference between the particles and the fluid were not published, and thus the particle radius is known only in some range. We assume the radius $0.13 \mathrm{~mm}$ corresponding to the mean radius of the particle range with the smallest radii. The estimates of $\phi_{\mathrm{cr}}$ and $\phi_{\text {eq }}$ are proportional to $r^{2}$ and can increase by a factor of about 3 if the largest particles were used. The

TABLE 1. Predicted Critical and Equilibrium Solid Fraction for the Experiments by Martin and Nokes [1988, 1989] (Cooling From Above and Heating From Below)

\begin{tabular}{crrcccccc}
\hline$R a$ & $P_{r}$ & $N u$ & $S$ & $w_{r m s}, \mathrm{~cm} / \mathrm{s}$ & $R e$ & $\phi_{\mathrm{cr}}$ & $\phi_{\text {eq }}$ & $\phi_{\text {eq }} / \phi_{0}$ \\
\hline $1.4 \times 10^{9}$ & 7 & 78 & $2.6 \times 10^{-1}$ & $3.6 \times 10^{-1}$ & 729 & $9.9 \times 10^{-2}$ & $5.2 \times 10^{-5}$ & 0.017 \\
$5.0 \times 10^{8}$ & 7 & 56 & $2.6 \times 10^{-1}$ & $2.3 \times 10^{-1}$ & 470 & $5.5 \times 10^{-2}$ & $3.2 \times 10^{-5}$ & 0.011 \\
$1.7 \times 10^{9}$ & 7 & 84 & $2.4 \times 10^{-1}$ & $4.0 \times 10^{-1}$ & 810 & $1.1 \times 10^{-1}$ & $6.3 \times 10^{-5}$ & 0.021 \\
$1.9 \times 10^{9}$ & 7 & 87 & $1.4 \times 10^{-1}$ & $4.1 \times 10^{-1}$ & 830 & $2.2 \times 10^{-1}$ & $2.0 \times 10^{-4}$ & 0.067 \\
$8.6 \times 10^{7}$ & 199 & 26 & $4.8 \times 10^{-2}$ & $1.8 \times 10^{-1}$ & 13 & $6.2 \times 10^{-2}$ & $1.1 \times 10^{-4}$ & 0.037 \\
$3.9 \times 10^{7}$ & 199 & 18 & $4.6 \times 10^{-2}$ & $1.9 \times 10^{-1}$ & 14 & $2.9 \times 10^{-2}$ & $3.7 \times 10^{-5}$ & 0.012 \\
$2.1 \times 10^{7}$ & 851 & 20 & $1.5 \times 10^{-2}$ & $1.4 \times 10^{-1}$ & 2.3 & $6.8 \times 10^{-2}$ & $4.1 \times 10^{-4}$ & 0.14 \\
$1.1 \times 10^{7}$ & 1890 & 22 & $1.9 \times 10^{-2}$ & $4.7 \times 10^{-2}$ & 0.35 & $8.2 \times 10^{-2}$ & $1.2 \times 10^{-3}$ & 0.4 \\
$5.1 \times 10^{6}$ & 4070 & 11 & $5.4 \times 10^{-2}$ & $8.0 \times 10^{-3}$ & 0.028 & $7.8 \times 10^{-2}$ & $1.2 \times 10^{-3}$ & 0.4 \\
\hline
\end{tabular}


thickness of the convective layer is $d=20 \mathrm{~cm}$. The initial volume fraction of particles $\phi_{0}$ (and approximately the initial mass fraction) is $\mathbf{0 . 0 0 3}$. In the case of $\operatorname{Pr}=\mathbf{7}$, the data on $N u$ and $w_{\mathrm{rms}}$ were not published, and we used Table A1 and Figure A1 of Martin and Nokes [1989] together with equations (A6) and (A8) to calculate $N u$ and $w_{\text {rms. }}$.

The efficiency factor $\epsilon$ has been calculated using the results of the experiment with reentrainment, where the equilibrium solid fraction was found to be about 0.4 of the initial fraction. Assuming that in this experiment the smallest particles were used, we calculated $\epsilon$ as follows:

$$
\epsilon=\frac{2 \Delta \rho^{2} g r^{2} c_{p} \phi_{\text {eq }}}{9 \alpha \eta_{0} F}=0.86 \% \text {. }
$$

It is interesting to note that this coefficient is close to the $0.6 \%$ value, which we have estimated earlier for the erosion of chemical layers using data by Olson [1984]. However, $\epsilon$ could be not constant as in the experiments by Olson [1984] and thus the estimated equilibrium solid fraction is very approximate.

The calculated theoretical parameters in Table 1 are the critical solid fraction $\phi_{c r}$ for an unimpeded sedimentation [Koyaguchi et al., 1990], the equilibrium solid fraction $\phi_{\text {eq }}$ estimated from our theory, and the ratio $\phi_{\mathrm{eq}} / \phi_{0}$, where $\phi_{0}$ is the initial solid fraction.

First of all, the initial solid fraction is well below the critical value $\phi_{\mathrm{er}}$ required for unimpeded sedimentation. Thus, the convection could not be suppressed by the suspended particles.

The equilibrium solid fraction is quite far below the initial solid fraction except for the last two experiments. The reentrainment was observed in the last experiment. Probably longer experiments are required to reach equilibrium in some other cases. However, it is more likely that in other experiments, reentrainment could not occur because the local mechanisms were not able to reentrain the particles, independently of our energetic criterion.

\section{Mechanism of SEDIMENTATION Suggested By KOYAGUCHI ET AL. [1990]}

Koyaguchi et al. [1990] found a regime of suspension where the system becomes unstable and an interface separating a lower sedimenting layer and almost particle free upper layer forms. A cyclic sedimentation was observed. This regime has been shown to occur already at a very small solid fraction, less than $1 \%$. Because these results could lead to a conclusion that sedimentation in magma oceans occurs already at very low degrees of crystallization, we consider here two questions: how does the criterion for sedimentation found by Koyaguchi et al. [1990] relate to our energetic criterion, and can it be applied to partially molten systems?

The criterion suggested by Koyaguchi et al. [1990] can be written for the solid fraction $\phi$ as follows:

$$
\phi>\phi_{\mathrm{cr}} \approx \frac{\rho \alpha \Delta T}{\Delta \rho} .
$$

This means that the thermally induced density perturbations $\alpha \Delta T$ becomes smaller than the density contrast $\phi \Delta \rho / \rho$ across the interface, where $\Delta \rho$ is the density difference between particles and the fluid.

The ratio of the equilibrium solid fraction $\phi_{\text {eq }}$ predicted from our theory to $\phi_{\mathrm{cr}}$ is

$$
\frac{\phi_{\mathrm{eq}}}{\phi_{\mathrm{cr}}}=\frac{\epsilon \kappa N u}{d u_{p}},
$$

where $u_{p}$ is the settling velocity. Thus, with increase of the intensity of convection $(N u)$, $\phi_{\text {eq }}$ exceeds $\phi_{c r}$ and the suspension could be controlled by the criterion ( 30$)$. For example, in the experiments by Martin and Nokes [1988, 1989] $d u_{p} / \kappa \sim 10$. For $\epsilon \sim 1 \%$ the criterion might be changed near $N u \sim 10^{3}$. This implies that at very high $N u$ the criterion of Koyaguchi et al. [1990] might separate not only the regimes of a gradual settling and the above unimpeded kind of settling but also separate the absolute suspension and this unimpeded settling.

To estimate the critical crystal fraction for a terrestrial magma ocean, we use the low-pressure values $\alpha \approx$ $5 \times 10^{-5} \mathrm{~K}^{-1}, \Delta \rho / \rho \approx 0.15, \Delta T \approx 500-1000 \mathrm{~K}$, and find

$$
\phi_{\mathrm{cr}} \sim \frac{\rho \alpha \Delta T}{\Delta \rho} \sim 0.25-0.50 .
$$

However, we suggest that the mechanism of settling cannot take place if the energetic conditions allow suspension. If the interface is formed, it can be eroded by the convection in the same way as in the compositionally layered systems [ $\mathrm{Ol}$ son, 1984] considered above. We have shown that both the suspension and erosion are controlled by similar energetic conditions and even the efficiency factors are similar. If the energetic criterion for the suspension is satisfied, then the interface must be eroded with the rate of settling or faster. The layers are remixed and the interface is not formed. Note that in this case the reentrainment mechanism is not connected with the reentrainment of particles but is essentially similar to the remixing in chemically layered systems studied by Olson [1984].

Consider now the case where the energetic criterion does not allow suspension and both mechanisms of settling can take place. In this case, realization of the mechanism of settling suggested by Koyaguchi et al. [1990] in partially molten systems could be difficult in the conditions of thermodynamical equilibrium. In a complete thermodynamical equilibrium, the crystal fraction varies with temperature according to the phase diagram and the effective thermal expansion (see detailed analysis by Solomatov and Stevenson [this issue (a)]) is due to not only the temperature changes but also due to the phase changes upon the melting. In a nonunivariant system, the effective thermal expansion is approximately equal to

$$
\alpha_{\mathrm{eft}}=\alpha+\frac{\Delta \rho}{\rho} \frac{d \phi}{d T} \approx \alpha+\frac{\Delta \rho}{\rho} \frac{1}{\Delta T_{m}} \approx \frac{\Delta \rho}{\rho} \frac{1}{\Delta T_{m}}
$$

where we assume a linear variation of the crystal fraction in the melting temperature range $\Delta T_{m}=T_{\text {liq }}-T_{\text {sol }}$ (between liquidus and solidus). We also used the fact that $\alpha_{\text {eff }} \gg \alpha$, meaning that in the melting range the density change due to the phase changes is larger than that due to the pure thermal expansion.

The criterion ( 30 ) is now written as

$$
\phi_{\text {cr }} \approx \frac{\Delta T}{\Delta T_{m}}<\frac{\bar{T}-T_{\text {sol }}}{\Delta T_{m}}=\phi(\bar{T}),
$$

where $\bar{T}$ is an averaged temperature of the well-mixed convective layer and $\phi(\bar{T})$ is the corresponding crystal fraction. Thus, in this linear approximation, the critical crystal fraction for the unimpeded sedimentation is always larger than the current value of the crystal fraction $\phi$ determined by the phase diagram. In other words, stronger buoyancy perturbations due to the increased crystal fraction in the cold boundary layers and the reduced crystal fraction in the hot 
boundary layers are comparable with the density contrast which would exist in the presence of an interface and prevent formation of such an interface. A completely disequilibrium partially molten system is similar to a simple suspension because the phase changes do not occur on the convective time scales.

We can estimate the equilibrium conditions from the requirement that the characteristic time scale spent by fluid elements in thermal boundary layers $t_{\text {conv }}$ is larger than the time scale $t_{\mathrm{ph}}$ for the phase transitions. The time scale $t_{\text {conv }}$ both for the laminar and turbulent convection is well-known [e.g., Kraichnan, 1962; Turcotte and Schubert, 1982]:

$$
t_{\mathrm{conv}} \sim \frac{\delta^{2}}{\kappa},
$$

where $\delta$ is the thermal boundary layer thickness.

In the simplest case, the rate-controlling factor for phase changes is diffusion (which could be the case in melting). In this case,

$$
t_{\mathrm{ph}} \sim \frac{r^{2}}{D}
$$

where $r$ is the crystal radius and $D$ is the diffusion coefficient for the melt. The equilibrium takes place if $t_{\text {conv }} \gg t_{\mathrm{ph}}$ or if

$$
r \ll \delta\left(\frac{D}{\kappa}\right)^{1 / 2} \sim 0.1 \delta
$$

where we used a two-phase equilibrium value for $\kappa \sim$ $10^{-3} \mathrm{~cm}^{2} \mathrm{~s}^{-1}$ and a typical value for $D \sim 10^{-5} \mathrm{~cm}^{2} \mathrm{~s}^{-1}$.

The thermal boundary layer thickness is estimated as

$$
\delta \approx \frac{d}{N u} \sim 10\left(\frac{\kappa k \eta}{\alpha g \rho F}\right)^{1 / 4} \sim 0.1-10 \mathrm{~cm}
$$

if $F=10^{5}-10^{9} \mathrm{ergs} \mathrm{cm}{ }^{-2} \mathrm{~s}^{-1}$, and $\eta=1-100 \mathrm{P}$. And thus the equilibrium takes place if $r \ll 10^{-2}-1 \mathrm{~cm}$.

Thus, the first suggestion is that in the case when the energetic criterion for the suspension does work, the regime found by Koyaguchi et al. [1990] cannot take place. The second conclusion is that in partially molten systems, the mechanism of sedimentation could be strongly controlled by the degree of thermodynamical equilibrium and in a complete equilibrium, the sedimentation mechanism found by Koyaguchi et al. [1990] might be impossible.

\section{Comparison With Rudman [1992]}

Rudman [1992] studied numerically two-dimensional, time-dependent, infinite Prandtl number two-phase convection, treating it with the help of averaged equations. At a given initial crystal fraction, three basic regimes are observed, depending on the ratio of crystal settling velocity to the maximum vertical convective velocity $S$, the crystal fraction $\phi$, and $\mathcal{F}=\rho \alpha \Delta T / \Delta \rho$ : complete settling, partial settling, and almost complete retention. The first regime occurs at high $\mathcal{F}$ and is similar to the regime found by $K o y$ aguchi et al. [1990]. The second regime is an intermediate one. The third regime corresponds to the regime described by Marsh and Maxey [1985] and Weinstein et al. [1988], where a retention region is formed near the upward flow of the convective cell. This regime can be called "kinematic": the settling does not take place because the particle trajectories are closed. The flow is steady and no mechanism of reentrainment of settled particles is involved. In a time- dependent convection (at high Rayleigh numbers) the particles scarcely can avoid escape from the retention regions (attractors in physical space are impossible, for example, in turbulent convection). In the absence of some reentrainment mechanism, all the particles would eventually settle down. Thus, we argue that suspension effects found in these experiments cannot be extrapolated to high Rayleigh number convection. We note that from the energetic point of view, the suspension cases studied by Rudman [1992] correspond to less than $0.01 \%$ of the maximum available power of the convection and that is about 2 orders of magnitude below the estimated efficiency factor in the experiments by Martin and Nokes [1988].

\section{Heat Flux From the Magma Ocean}

The heat flux is an essential parameter for suspension. The heat flux from the magma ocean is determined by convection in the magma ocean and by the radiative properties of the surface. We use the simplest assumption that the atmosphere chemistry depends only on the surface temperature but not on the potential temperature, that is, the temperature just beneath the surface thermal boundary layer. (As in meteorology, the "potential temperature" is a convenient way of labeling adiabats in a system that is mostly adiabatic. We choose to define it as the actual temperature just below the thermal boundary layer.) In this case the heat flux at a given surface temperature can be calculated with the help of a blackbody model or a greenhouse model [Zahnle et al., 1988]. The heat flux in the blackbody model is

$$
F=\sigma T^{4},
$$

where $\sigma=5.67 \times 10^{-5} \mathrm{ergs} \mathrm{cm}^{-2} \mathrm{~K}^{-4}$ is the StefanBoltzmann constant. In the case of a steam atmosphere the results of calculations by Zahnle et al. [1988] are parameterized with the help of a simple function:

$$
F=1.5 \times \times^{5}+1.02 \times 10^{-2} \exp \left(0.011 T_{s}\right), \mathrm{ergs} \mathrm{cm}^{-2} \mathrm{~s}^{-1} \text {. }
$$

This parameterization is accurate for $500<T_{s}<1600 \mathrm{~K}$. At higher temperatures (possibly up to about $2000 \mathrm{~K}$ ) it can be used as an extrapolation.

The surface boundary condition requires that the same heat flux is transported by the convection in the magma ocean. In the case of the turbulent convection the heat flux is equal to

$$
F=a\left(T-T_{\theta}\right)^{4 / 3}\left(\frac{\alpha g \rho c_{p} k^{2}}{\nu}\right)^{1 / 3},
$$

where $T$ is the potential temperature, $k$ is the thermal conductivity, $\nu$ is the kinematic viscosity, and the coefficient $a \approx \mathbf{0 . 0 8 9}$ is for the free boundary case [Kraichnan, 1962] and not very different for other cases [e.g., Garon and Goldstein, 1973; Busse, 1978].

Some problems occur due to strong variations of the parameters with temperature and pressure. We assume that in the case of any strong variation of the parameters with depth, only the surface layer of the order of the Kolmogorov length scale (meters) is important for the thermal boundary layer calculations. This is an obvious assumption in classical thermal turbulence because the scaling of the turbulent convection does not involve depth dependence. We note, however, that a weak dependence on depth could arise in 
the hard turbulence regime [Heslot et al., 1987; Castaing et al., 1989].

In the case of two-phase flow an effective thermal capacity and thermal expansion should be used; they are about 1 order of magnitude larger than their one-phase values [Solomatov and Stevenson, this issue (a)]. We will use the values $\alpha=10^{-3} \mathrm{~K}^{-1}$ and $c_{p}=10^{8} \mathrm{ergsg}^{-1} \mathrm{~K}^{-1}$. The large viscosity contrasts together with disequilibrinm effects produce much larger uncertainties. In particular, due to highly disequilibrium conditions in the thermal boundary layers, even at low surface temperatures (e.g., below the solidus) the crystal fraction can still be the same as below the thermal boundary layer (e.g., zero). This means that the kinetics is too slow for the crystals to nucleate and grow. The problem was discussed earlier in the case of melting (see (36)-(38)). Crystallization could be a much slower process because the rate-controlling mechanism is interface kinetics, possibly surface nucleation [Solomatov and Stevenson, this issue (b)]. Even in the absence of this effect, the influence of a thin (centimeters) highly viscous layer on the turbulent or laminar convection is poorly understood.

Thus, two limiting cases will be considered. In the first case there is no effect of the high-viscosity surface sublayer, and (41) is applied without any changes. In the second case we assume that below some critical temperature corresponding to about 0.6 crystal fraction, the suspension becomes quasi-solid and this surface solidlike region effectively does not participate in the convection. The equation for the heat flux is then

$$
F=a\left(T-T_{\mathrm{rh}}\right)^{4 / 3}\left(\frac{\alpha g \rho c_{p} k^{2}}{\nu}\right)^{1 / 3},
$$

where $T_{\mathrm{rh}}$ is the temperature at the rheological boundary.

The results of the calculations for these cases are shown in Figures $1 a$ and $1 b$. According to simplified calculations of the phase diagram and the adiabats (Figure 2), the temperature range considered $(<2000 \mathrm{~K})$ corresponds to the solidification of the upper mantle. Note that the adiabats are valid only in the low-viscosity regions and do not represent the superadiabatic temperature distribution below the critical crystal fraction, where the viscosity rapidly increases [Solomatov and Stevenson, this issue (a)].

When the potential temperature drops just below the liquidus, the heat flux is estimated as $10^{7}-10^{8}$ ergs cm $^{-2} \mathrm{~s}^{-1}$ because of low viscosity. With the temperature drop, the heat flux decreases due to both the surface temperature drop and also to the magma viscosity increase. However, the heat flux drops significantly only when the potential temperature is near the critical temperature where the crystal fraction is about 0.6. This means that a large heat flux is maintained until the high-viscosity bottom of the magma ocean almost reaches the surface, leaving only a global shallow "magma chamber".

During solidification of the lower mantle, the potential temperature is certainly higher than $2000 \mathrm{~K}$ (Figure 2). Thompson and Stevenson [1988] suggest that in this case the atmosphere is a "silicate" one because the silicates partially vaporize. The heat flux is then estimated to be $10^{8}-10^{9}$ ergs $\mathrm{cm}^{-2} \mathrm{~s}^{-1}$.

\section{Critical Crystal Radius for Suspension in a Magma OCEAN}

The critical crystal radius is defined by ( 18) with $\epsilon=1$ and with $D=1$. This corresponds to the condition that the
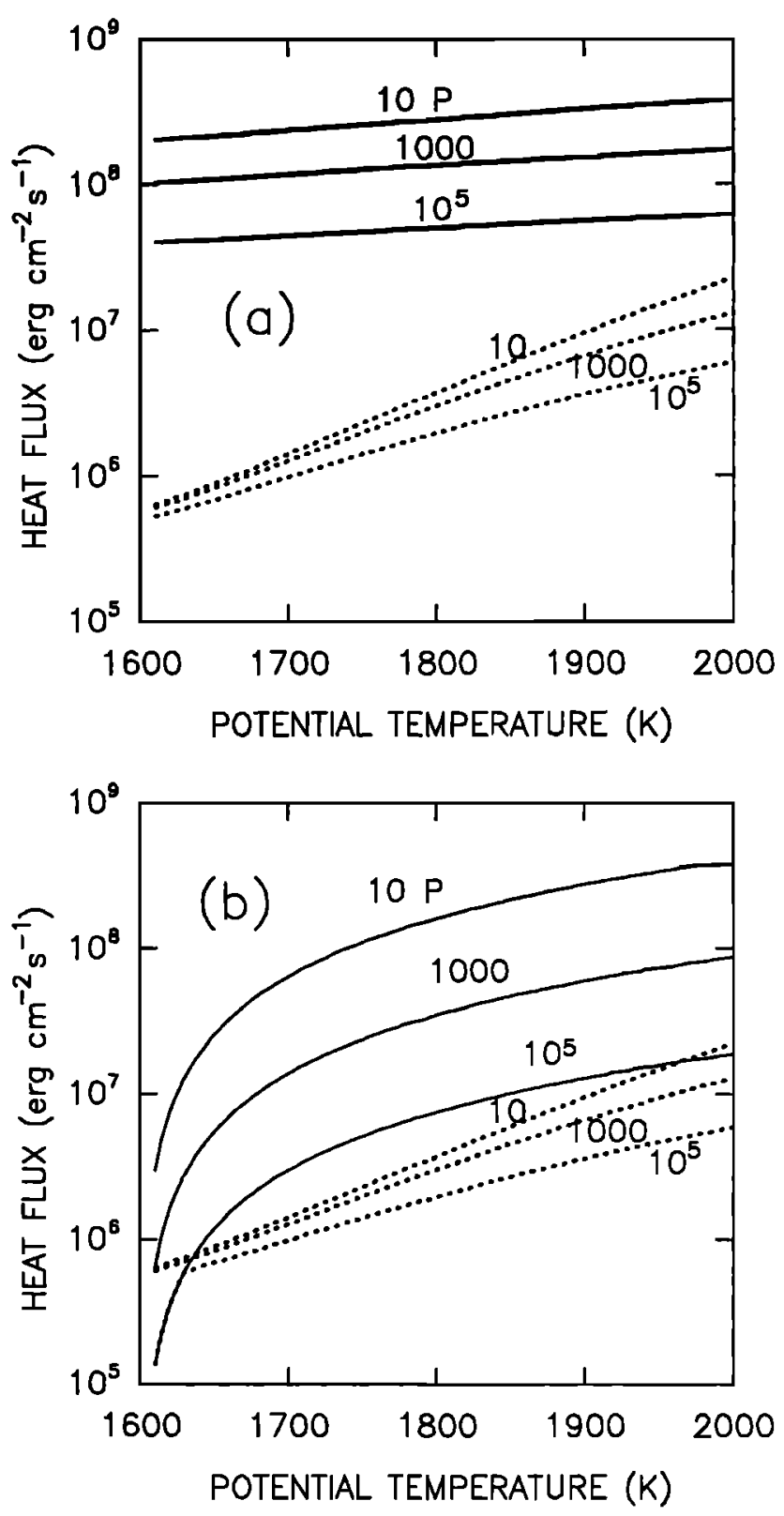

Fig. 1. Calculation of the heat flux from the magma ocean under different assumptions: (a) convection occurs effectively without any rheological boundary layer; $(b)$ a surface layer with the temperature less than $1600 \mathrm{~K}$ has much higher viscosity and effectively does not participate in the convection. Solid lines correspond to the blackbody boundary conditions, and the dotted lines to a steam atmosphere. The viscosity of the crystal-melt suspension labels the curves.

heat production due to settling is equal to the heat loss from the magma ocean. Thus, this is a maximum crystal radius above which sedimentation and fractional differentiation are unavoidable. The crystal radius required for suspension is a fraction $(\epsilon D)^{1 / 2}$ of this critical radius. This fraction is equal to $\left.(\epsilon D)^{1}\right\rangle_{2} \approx 0.1$, if $\epsilon \approx 1 \%$ and $D \approx 1$.

Figure 3 shows the dependence of the critical crystal radius on the heat flux and the crystal fraction for a fixed viscosity of the melt ( $1 \mathrm{P}$ ). The calculation of the settling velocity of the particles takes into account three different dependences on the particle Reynolds number in Stokes, Newton, and transition regimes [Bird et al., 1960] and de- 


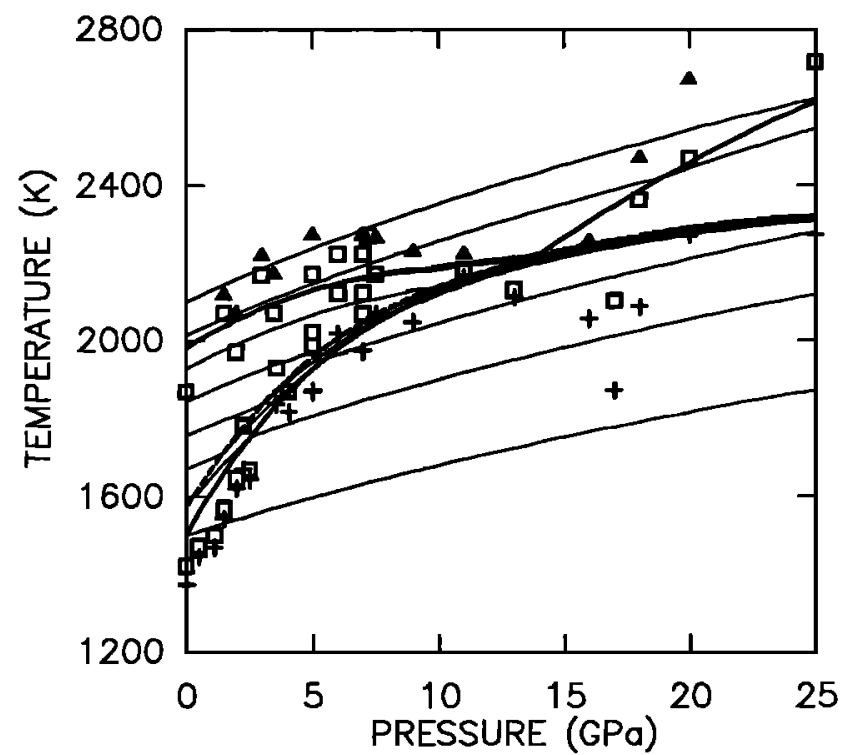

Fig. 2. From Solomatov and St evenson [this issue (a), Figure $3 b$ ). The solidus, liquidus, temperature of the second phase appearance (dashed line), and adiabats are shown for the upper mantle region together with the experimental data for peridotites. Liquid region is marked with triangles, solid region with crosses and the partially molten region with squares. The first liquidus phase is olivine at $p<13.7 \mathrm{GPa}$ and gamet at $p>13.7 \mathrm{GPa}$. Potential temperature is defined as the surface values of the adiabats.

pendence of the crystal fraction according to equation (13). The local criterion requiring that the frictional velocity must be larger than the convective velocity is also shown. The calculations for the turbulent boundary layer were made as in the case of shear turbulence [Kraichnan, 1962; Landau and Lifshits, 1989] and correspond to the calculations done by Tonks and Melosh [1990]. The crystal fraction is assumed to be distributed uniformly. The lowest of the values predicted

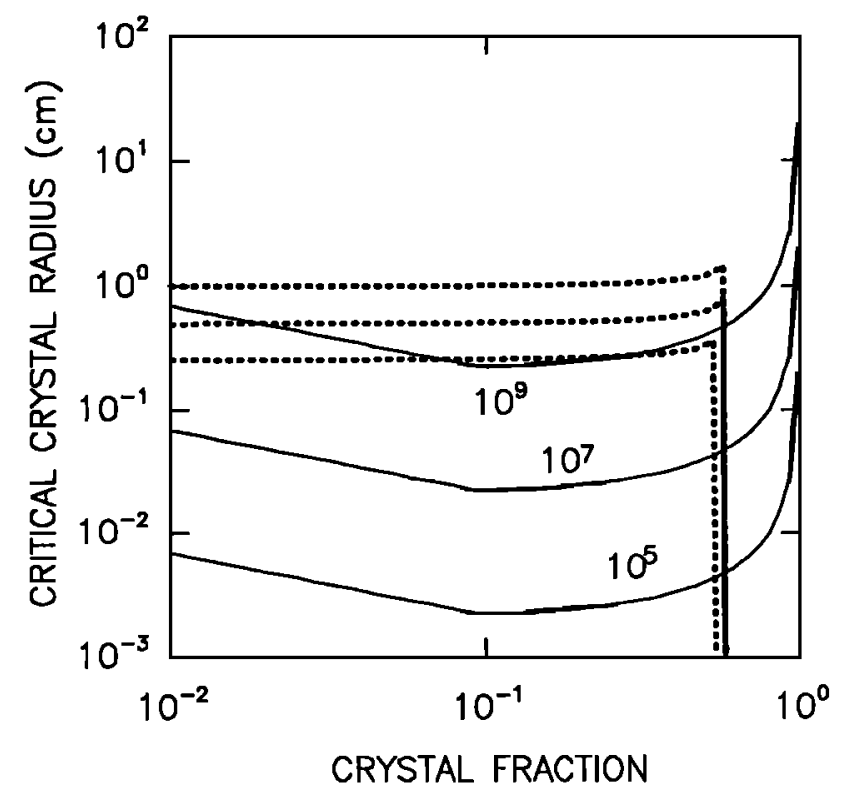

Fig. 3. The dependence of the critical crystal radius for suspension on the crystal fraction for different values of the heat flux: $10^{9}, 10^{7}$ and $10^{5}$ ergs cm ${ }^{-2} \mathrm{~s}^{-1}$. The critical radius for the local criterion for suspension in a turbulent layer is also shown (dotted lines). The viscosity is $1 \mathrm{P}$, the density difference between the crystals and the melt $\Delta \rho / \rho=0.1$. by the energetic and local criteria determines the critical crystal radius. The energetic criterion predicts much lower values in the most important crystal fraction range. Above about $10 \%$ crystal fraction the critical radius weakly depends on the crystal fraction until a sharp transition due to the viscosity change near the maximum packing crystal fraction occurs (we used the Roscoe equation for the viscosity; see Solomatov and Stevenson [this issue (a)] for discussion, and 0.6 as the maximum crystal fraction). This transition changes the dynamical regime (from turbulent to laminar convection) but does not influence the energetic conditions at a given heat flux. So the greatest risk for the collapse of suspension occurs near $10 \%$ crystal fraction.

In the magma ocean, the equilibrium crystal fraction in the absence of settling changes continuously from zero at the pressure where adiabat intercepts the liquidus to some maximum value at the bottom. The characteristic depth for the crystal fraction change is comparable or even larger than the thickness of the magma ocean [Solomatov and Stevenson, this issue (a)] From Figure 3 we find that the change in the critical crystal radius with crystal fraction and thus with the depth is very small in comparison with the dependence on all other factors and with possible uncertainties in the crystal radius. To prevent fractional crystallization, it is sufficient to require that at about $10 \%$ of the total crystal fraction in the magma ocean the criterion is satisfied.

Figure 4 shows the results of calculations of the critical crystal radius for suspension as a function of the heat flux and the viscosity of the melt at the critical crystal fraction $10 \%$. The calculations take into account different regimes of crystal settling depending on the Reynolds number for the settling particles [Bird et al., 1960; Soo, 1967] Using the calculations of the heat flux, we take $F \sim 10^{8}-$ $10^{9}$ ergs $\mathrm{cm}^{-2} \mathrm{~s}^{-1}$ when the adiabat intersects the liquidus at high pressures $p>15 \mathrm{GPa}$ and $F \sim 10^{6}-10^{8} \mathrm{ergs} \mathrm{cm}^{-2} \mathrm{~s}^{-1}$ for $p<15 \mathrm{GPa}$. The viscosity is also an uncertain parameter. At the zero-pressure liquidus (about $2000 \mathrm{~K}$ ), the viscosity of molten ultramafic silicates (peridotite or chondrite

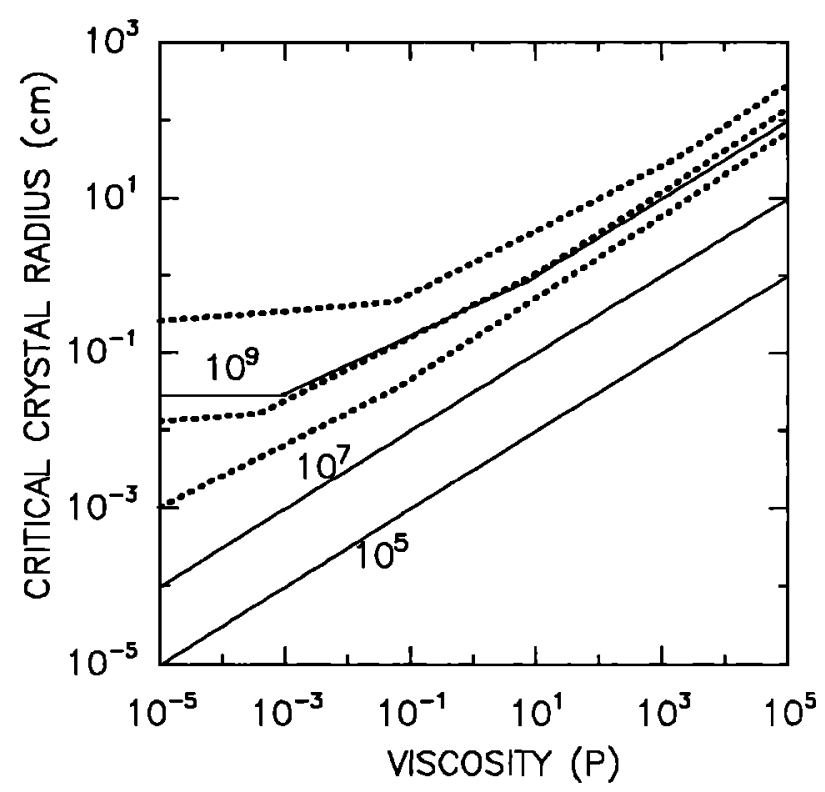

Fig. 4. The dependence of the critical crystal radius for suspension on the viscosity of the melt for different values of the heat flux at the extremum crystal fraction $10 \%$. 
compositions) can be estimated with methods suggested by Bottinga and Weill [1972], Shaw [1972], or, recently, Persikov et al. [1990]. The estimates are about $\eta \sim 1 \mathrm{P}$ depending slightly on the composition (mostly on the $\mathrm{SiO}_{2}$ content). Due to structural changes, the viscosity of most silicates tends to decrease with pressures up to a pressure of about $25 \mathrm{GPa}$ where all major structural changes are completed [Kushiro, 1980; 1986; Persikov et al., 1990]. The viscosity along the liquidus or at a constant temperature drops by several orders of magnitude for high $\mathrm{Al}$ and $\mathrm{Si}$ melts because they suffer a significant depolymerization with pressure. The ultramafic melts are polymerized to a much lower degree already at small pressures and thus, their viscosity must drop less rapidly [Kushiro, 1986] although quantitative data are absent. However, molecular dynamic simulations of $\mathrm{MgO}-\mathrm{SiO}_{2}$ by $\mathrm{E}$. A. Wasserman et al. (Compositional dependences of transport and thermodynamic properties of $\mathrm{MgO}-\mathrm{SiO}_{2}$ mixture by molecular dynamics, submitted to Journal of Geophysical Reseach, 1992) give an estimate for the viscosity expected in essentially depolymerized melts $10^{-2}-10^{-1} \mathrm{P}$ at $5 \mathrm{GPa}$. This value is almost independent of temperature and composition and is close to an absolute minimum value [Persikov et al., 1990]. The viscosity of a completely depolymerized melt increases with pressure. This increase is not big in comparison with the changes due to structural transformations and can be roughly estimated with the approach suggested by Andrade [1952] and applied to the Earth's core by Gans [1972]. This approach supposes that the viscosity is inversely proportional to the distance between the molecules and proportional to the solidlike Debay or Einstein characteristic frequency. As a result, the viscosity varies with density as $\rho^{\gamma+1 / 3}$, where $\gamma=1-2$ is the Grüneisen parameter. An increase within 1 order of magnitude can be expected in the entire magma ocean pressure range. Thus, the viscosity of the magma ocean near the liquidus is estimated as $10^{-2}-1 \mathrm{P}$.

During crystallization at high pressures, the viscosity of a depolymerized melt does not necessarily increase due to compositional changes as at lower pressures although, of course, it increases due to the temperature drop. Moreover, in some pressure ranges the first liquidus phase is a low $\mathrm{SiO}_{2}$ phase (majorite garnet and possibly magnesiowüstite [Solomatov and Stevenson, this issue (a)]) that at (hypothetical) low-pressure conditions would result even in a decrease in the viscosity of the coexisting melt.

Summarizing estimates for the heat flux and the viscosity, we conclude that the critical radius of the crystals is $10^{-2}-1 \mathrm{~cm}$ for $p>15 \mathrm{GPa}$ where the heat flux is large $\left(10^{8}-19^{9}\right.$ ergs cm $\left.{ }^{-2} s^{-1}\right)$, and $10^{-3}-10^{-1} \mathrm{~cm}$ for $p<15$ GPa where it is somewhat less $\left(10^{7}-10^{8} \mathrm{ergs} \mathrm{cm}^{-2} \mathrm{~s}^{-1}\right)$. Sedimentation must occur for larger crystals. The absolute suspension as we discussed is possible for crystal radii about one order of magnitude smaller: $10^{-3}-10^{-1} \mathrm{~cm}$ for $p>15$ $\mathrm{GPa}$ and $10^{-4}-10^{-2} \mathrm{~cm}$ for $p<15 \mathrm{GPa}$. Partial sedimentation takes place in between.

These estimates for the critical crystal size are significantly smaller (by several orders of magnitude) than all previous estimates [Tonks and Melosh, 1990; Miller et al., $1991 a, b]$. Thus, the problem of crystal sizes becomes one of the central problem of magma oceans. It is discussed by Solomatov and Stevenson [this issue (b)]. Other uncertainties come from the unknown dependence of the viscosity on pressure, and the uncertain boundary conditions determining the heat flux from the magma ocean.

\section{Laminar-TURBUlENT Transition}

Even if the energetic conditions allow a suspension, some local mechanisms must reentrain the particles from the bottom. Turbulence (dynamical turbulence) can be considered as a possible mechanism of re-entrainment. The criterion that the frictional velocity in the turbulent boundary layer must be approximately larger than the settling velocity is important for the shear turbulence [e.g., Soo, 1967] and possibly is applicable to the convective turbulence as was suggested by Tonks and Melosh [1990]. In this and the following sections we discuss a part of this problem, laminar-turbulent transition in a magma ocean.

Developed turbulent convection is usually described in terms of mixing length theory [Priestly, 1959; Kraichnan, 1962; Clayton, 1968; Golitsyn, 1978] which predicts the following dependence of the convective velocity fluctuations on the mixing length $l_{0}$ :

$$
u_{0} \approx\left(\frac{\alpha g l_{0}}{c_{p}} \frac{F}{\rho}\right)^{1 / 3}
$$

The convective velocities for smaller length scales $l$ are found from the Kolmogorov cascade law $u \sim u_{0}\left(l / l_{0}\right)^{1 / 3}$. The smallest length scale is the Kolmogorov length scale for which the Reynolds number is about the critical one $R e_{\mathrm{cr}}$. The transition from turbulent to laminar convection occurs approximately when the Kolmogorov length scale becomes equal to the mixing length and then the inertial range of convection vanishes.

The viscosity at which the turbulence in the magma ocean vanishes is

$$
\eta_{\mathrm{tr}} \approx\left(\frac{\alpha g \rho^{2} l_{0}^{4} F}{A c_{p}}\right)^{\frac{1}{3}} \approx \frac{\rho d}{R e_{c \mathrm{r}}}\left(\frac{\alpha g l_{0}}{c_{p}} \frac{F}{\rho}\right)^{1 / 3}
$$

[Kraichnan, 1962]. The second equation shows that at the transition the Reynolds number for the convective flow is simply equal to $R e_{\mathrm{cr}}$.

Some modification of this criterion is needed due to the multiphase medium of the magma ocean. In accordance with Solomatov and Stevenson [this issue $(a)$ ] the equilibrium thermodynamics of the magma ocean can be described in terms of effective thermodynamical parameters. The mixing length $l_{0}$ is of the order of the thickness $d$ of the convective layer or of the temperature scale height $c_{p} / \alpha g$ if it is smaller than the thickness of the layer. In the case of the one-phase magma ocean, $c_{p} / \alpha g d \approx 1-10$ and so $l \approx d$. In the case of multiphase system, the ratio $c_{p} / \alpha g$ cannot change by more than a factor which is equal to the ratio of the melting gradient to the adiabatic gradient and is about $1-3$, depending on pressure. As a consequence, the velocities cannot increase by more than a factor of about 12 due to the equilibrium phase transitions. The estimate $c_{p} / \alpha g d \approx 0.1-1$ is a reasonable one for both a one-phase and multiphase magma ocean.

Using typical values of the parameters $\alpha g d / c_{p} \approx 0.1-$

$1, \rho \approx 4 \mathrm{~g} \mathrm{~cm}^{-3}, \quad d \approx 10^{8} \mathrm{~cm}, \quad F \approx 10^{6}-$ $10^{9} \mathrm{ergs}^{-2} \mathrm{sm}^{-1}, R e_{\mathrm{cr}} \approx 30$, we obtain

$$
\eta_{\mathrm{tr}} \approx 10^{9}-10^{10} \mathrm{P} \text {. }
$$

We see that the transition from turbulent to laminar convection occurs when the magma ocean solidifies significantly (up to approximately the maximum packing crystal fraction [Solomatov and Stevenson, this issue (a)]. 
Because the transition from turbulent to laminar convection can be sensitive to such factors as rotation, nonNewtonian rheology, and density gradient due to sedimentation we estimate these effects in subsequent sections. The kinetic effects (disequilibrium between the crystals and melt) are also important, but they are not considered here.

\section{INFLUENCE OF ROTATION ON THE CONVECTION IN A MAGMA OCEAN}

Convection in magma oceans occurs in the presence of rotation. This effect is very important, for example, for the dynamics of stars and giant planets and could be also important for the magma oceans. The main physical effect of the rotation is the presence of the Coriolis force. The additional nondimensional parameter is the Taylor number,

$$
T a=\frac{4 \Omega^{2} d^{4}}{\nu^{2}}
$$

where $\Omega$ is the angular velocity, $d$ is the thickness of the layer and $\nu$ is the kinematic viscosity. The Taylor number expresses the square of the ratio between the Coriolis force and the viscous force. The relative importance of the inertia and the Coriolis forces is described by the Rossby number

$$
R_{0}=\frac{u_{0}}{2 \Omega d}
$$

where $u_{0}$ is a characteristic velocity of the flow.

The linear regime has been studied by Chandrasekhar [1953, 1961], Nakagawa and Frenzen [1955], and in many subsequent experiments. Boubnov and Golitsyn [1986, 1990], and Golitsyn [1990] distinguish several transitions in the convective regimes: from linear to nonlinear regime, from regular to irregular regime, and from geostrophic to non-geostrophic regime. All transitions, especially the first two, are poorly measured and identified. In the irregular regime the velocity measurements [Boubnov and Golitsyn, 1986, 1990; Chen et al., 1989; Fernando et al., 1991] justify the formula obtained by Golitsyn [1980, 1981] for the characteristic velocity of the convective flow:

$$
u_{0} \sim\left(\frac{\alpha g F}{\rho c_{p} \Omega}\right)^{1 / 2} \sim \frac{\kappa}{d} R a_{f}^{1 / 2} T a^{-1 / 4}
$$

where

$$
R a_{f}=\frac{\alpha g F d^{4}}{k \kappa \nu}
$$

is the Rayleigh number based on the heat flux.

The distance between vortices in the observed turbulent flow is a subject of discussion [Dikarev, 1983; Boubnov and Golitsyn, 1986; Chen et al., 1989]. Possibly more accurate measurements were done in the experiments by Hopfinger et al. [1982] and Hopfinger [1989] on turbulence produced by an oscillating grid in a rotating container. They suggested that the largest space scale of the turbulent motion in a rotating system is determined by the balance between Coriolis and inertia forces.

If such a characteristic space scale does exist it would be reasonable to treat it as the largest inertial scale of the turbulent motion and thus to use it as the scale for the mixing length. So, we assume that the largest size of turbulent eddies $l_{0}$ is determined by the size for which the Coriolis force is equal to the inertia force (the Rossby number determined for this scale is equal to 1 ):

$$
R o\left(l_{0}\right) \sim \frac{u_{0}}{\Omega l_{0}} \sim 1 .
$$

An anisotropy of the turbulent flow can be important [ $\mathrm{Cabot}$ et al., 1987], but following the experiments by Fernando et al. [1991] we assume that the velocities in all directions are of the same order of magnitude despite an anisotropic flow structure.

Consider the consequences of this simple interpretation. When the Rossby number calculated for the size of the system exceeds unity, the "geostrophic turbulence" is changed by the "normal" turbulence which does not feel the rotation. The transition occurs at

$$
\operatorname{Ra}_{f} \sim \operatorname{Pr}^{2} \operatorname{Ta}^{3 / 2} \text {. }
$$

This equation coincides with the equation suggested by Boubnov and Golitsyn [1990].

Equation (48) is also obtained immediately with the help of (43) for the turbulent velocity. The size of the largest eddies is found as follows

$$
l_{0} \sim \frac{u_{0}}{\Omega} \sim\left(\frac{\alpha g F}{\rho c_{p} \Omega^{3}}\right)^{1 / 2} \sim d \operatorname{Ra}_{f}^{1 / 2} \operatorname{Pr}^{-1} \mathrm{Ta}^{-3 / 4}
$$

In accordance with the mixing length theory the temperature fluctuation scale is equal to

$$
\delta T \sim\left(\frac{F^{2}}{\alpha \rho^{2} g c_{p}^{2} l_{0}}\right)^{1 / 3} \sim\left(\frac{F \Omega}{\alpha \rho g c_{p}}\right)^{1 / 2} .
$$

This dependence was suggested by Boubnov and Golitsyn [1990] and was supported by their experiments and those of Fernando et al. [1991].

So the simple scaling based on the mixing length theory results in a good agreement with the experiments. The basic scaling laws for the "geostrophic turbulence" are summarized in the following form:

$$
\begin{gathered}
u_{0} \sim u_{0}(0) R o_{f}^{1 / 2}, \\
l_{0} \sim l_{0}(0) R o_{f}^{3 / 2}, \\
\delta T \sim \delta T(0) R o_{f}^{-1 / 2},
\end{gathered}
$$

where $u_{0}(0), l_{0}(0)$, and $\delta T(0)$ are the characteristic velocity, size and temperature fluctuation for the largest eddies in the absence of rotation, and

$$
R o_{f}=\left(\frac{\alpha g F}{\rho c_{p} d^{2} \Omega^{3}}\right)^{1 / 2} \sim \frac{u_{0}}{d \Omega}
$$

is the Rossby number based on the heat flux (on the velocity in the absence of rotation).

The turbulence ceases when the inertial interval, between the largest turbulent scale $l_{0}$ and the Kolmogorov scale

$$
l_{K} \sim\left(\frac{\nu^{3} \rho c_{p}}{\alpha g F}\right)^{1 / 4},
$$

disappears, and thus $l_{0} \sim l_{K}$. We obtain

$$
R a_{f} \sim \operatorname{Pr}^{2} T a \text {. }
$$

In Figure 5 we summarize our qualitative results, plotting the convective diagram for the parameter range corresponding to the experimental data. Possibly, the boundary between the regular and irregular convective regimes determined with large uncertainties in the experiments by Boubnov and Golitsyn [1986, 1990] corresponds to the transition 


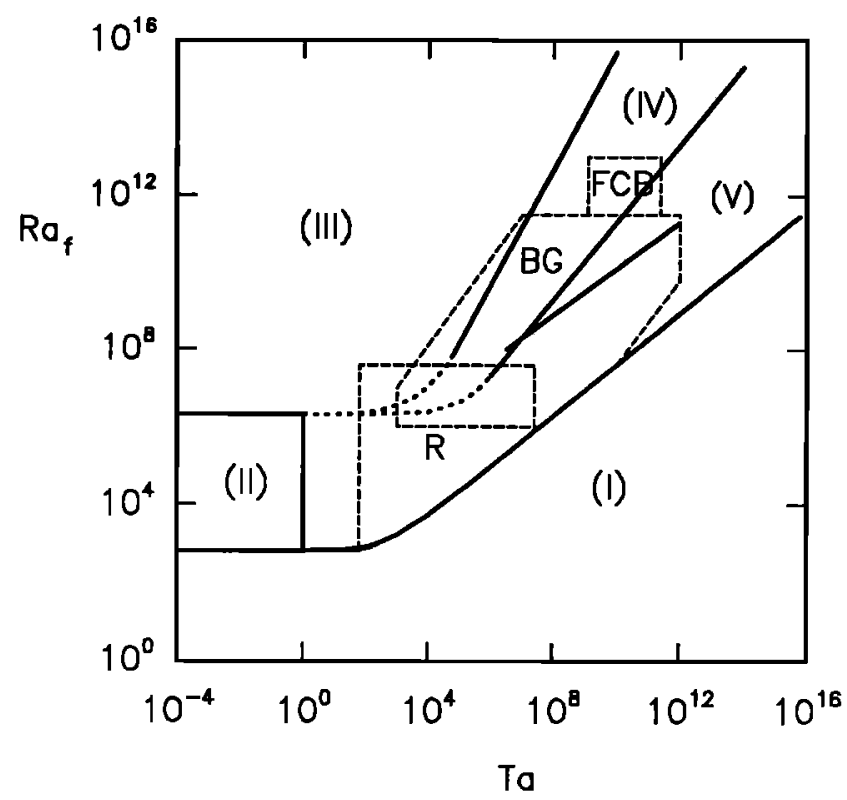

Fig. 5. An approximate diagram for the convective regimes in the presence of rotation in $T a-R a_{f}$ parameter space for $P T=4.5$ : (I) conductive regime; (II) laminar convection without any effect of the rotation, (III) turbulent convection without any effect of the rotation; (IV) "geostrophic turbulence"; (V) "geostrophic laminar convection". The transitions are separated by the solid lines corresponding to asymptotic dependences. Dotted lines are possible transitions between the asymptotic curves. The short solid line is a transition from regular to irregular convection suggested by Boubnov and Golitsyn [1990] which could be identified with the suggested transition between the laminar (V) and turbulent (IV) geostrophic convection. The regions of experimental studies are delineated by dashed lines: (R) Rossby [1969]; (BG) Boubnov and Golitsyn [1986, 1990]; (FGB) Fernando et al. [1991]. The stability boundary was calculated by Chandrasekhar [1953], the transition (II)-(III) from laminar to turbulent convection was estimated from Kraichnan's [1962] work. The boundary between the regions (III) and (IV) was suggested by Boubnov and Golitsyn [1990].

between laminar and turbulent geostrophic convection suggested above. However, we find that this boundary is not parallel to the stability curve as suggested by Boubnov and Golitsyn [1986, 1990] (see also Golitsyn [1991]). It has a slope larger than the stability curve but smaller than the slope of the transition to non-geostrophic regime.

In the case of a totally molten magma ocean we assume $\Omega \approx 10^{-4} \mathrm{~s}^{-1}, d \approx 10^{8} \mathrm{~cm}, \nu \approx 1 \mathrm{~cm}^{2} \mathrm{~s}^{-1}, F \approx$ $10^{9}$ ergs cm ${ }^{-2} \mathrm{~s}^{-1}, \kappa \approx 10^{-2} \mathrm{~cm}^{2} \mathrm{~s}^{-1}$ (previous sections), and find that

$$
R a_{f} \sim 10^{36}, \quad \operatorname{Pr} \sim 10^{2}, \quad T a \sim 10^{25}, \quad R o_{f} \sim 10^{-2} .
$$

For the initial stage of the evolution we find

$$
\begin{gathered}
u_{0} \sim 10^{-1} u_{0}(0) \sim 10^{2} \mathrm{~cm} \mathrm{~s}^{-1}, \\
l_{0} \sim 10^{-3} d \sim 10^{5} \mathrm{~cm}, \\
\delta T \sim 10 \delta T(0) \sim 1 \mathrm{~K} .
\end{gathered}
$$

The correction due to rotation is even larger (a factor of $\sim$ $1 / 2$ for the velocity and an order of magnitude for the mixing length) if the rotation of the magma ocean was several times faster than today [e.g., Lambeck, 1980].

Figures $6 a$ and $6 b$ show the main transition curves, initial position of the magma ocean and its position in the case of a continuous increase of the viscosity of a magma ocean at the same thickness of the convective layer (Figure $6 a$ ) and in the case of a continuous decrease of the thickness of the magma ocean at the same viscosity (Figure $6 b$ ). Because of the crystal fraction variation with depth, the magma ocean consists of a low-viscosity upper layer (its thickness significantly drops during crystallization) and a high-viscosity lower layer (its viscosity significantly increases during crystallization) and roughly corresponds to Figure $6 b$ and Figure $6 a$. It is, of course, a simplified representation of a highly non-uniform magma ocean.

Summarizing the results, we see that the velocity of the convective flow is reduced by one order of magnitude due to rotation and that the transition from turbulent to laminar flow requires still a large increase in the viscosity and occurs near the maximum packing crystal fraction [Solomatov and Stevenson, this issue $(a)$ ].
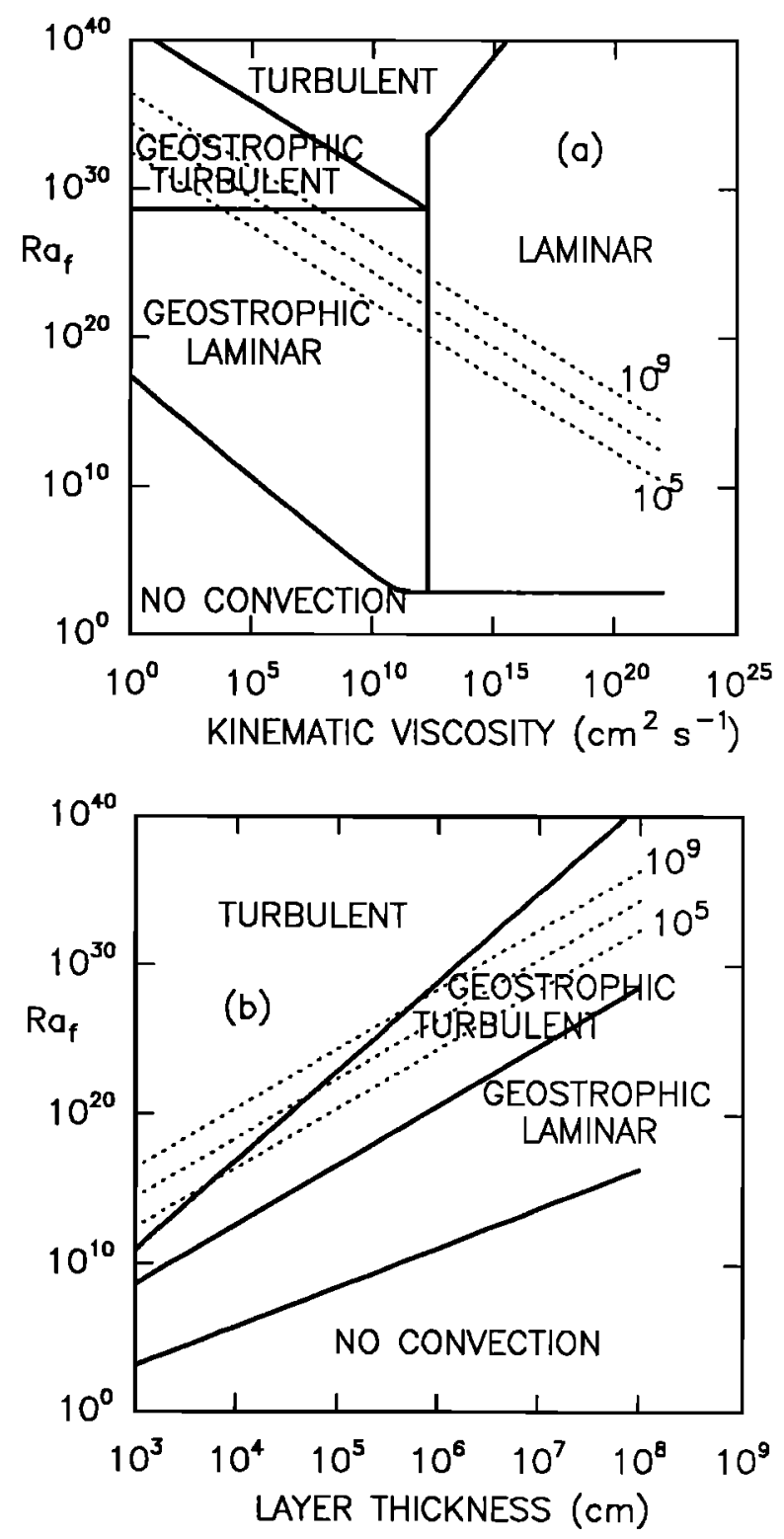

Fig. 6. Approximate calculations of the position of a magma ocean in the parameter space $\nu-R a_{f}$ for the heat fluxes $10^{5}, 10^{7}$ and $10^{9} \mathrm{ergs} \mathrm{cm}^{-2} \mathrm{~s}^{-1}:$ (a) the viscosity changes at a fixed depth of the layer $1000 \mathrm{~km} ;(b)$ the depth of the layer changes at a fixed viscosity $1 \mathrm{~cm}^{2} \mathrm{~s}^{-1}$. 
INFLUENCE OF NON-NEWTONIAN RHEOLOGY ON THE Transition from Turbulent to Laminar Convection IN A Magma OCEan

A non-Newtonian behavior of magmas was observed in various experiments [Robson, 1967; Shaw et al., 1968; Shaw, 1969; Pinkerton and Sparks, 1978; McBirney and Murase, 1984; Ryerson et al., 1988]. Some physical interpretation was suggested by Solomatov and Stevenson [1991]. In this section we estimate the influence of this factor on the transition from turbulent to laminar convection in the magma ocean.

\section{Basic Assumptions}

We assume that the transition from turbulent to laminar convection is determined by the condition

$$
\frac{\rho u_{0}^{2}}{\tau_{0}} \sim R e_{c r},
$$

where the subscript zero refers to the largest turbulent scale. This condition has the same physical meaning as in the constant viscosity case: a balance between the inertia $\left(\rho u_{0}^{2}\right)$ and the viscous $\left(\tau_{0}\right)$ forces on the largest convective scale (see also Ryan and Johnson [1959]). The inertia range of turbulent convection and thus the turbulent velocity scale (43) does not depend on the rheology.

The effective shear stress $\tau_{0}$ is estimated from the rheological law:

$$
\tau_{0} \sim \tau\left(\dot{e}_{0}\right)
$$

where

$$
\dot{e}_{0} \sim \frac{u_{0}}{l_{0}}
$$

is the characteristic strain rate at the transition.

\section{Power Law Viscosity}

We consider the rheological law in the form

$$
\tau_{i j}=b \frac{\dot{e}_{i j}}{\dot{e}^{1-\beta}}
$$

where $\dot{e}$ is the second invariant of the strain rate tensor, $b$, and $\beta<1$ are constants. The case $\beta=1$ corresponds to the constant viscosity case.

The characteristic shear stress at the transition can be written as

$$
\tau_{0} \sim \tau_{R}\left(\frac{\dot{e}_{0}}{\dot{e}_{R}}\right)^{\beta} \sim \eta_{R} \dot{e}_{R}\left(\frac{u_{0}}{l_{0} \dot{e}_{R}}\right)^{\beta},
$$

where $\tau_{R}, \dot{e}_{R}$, and $\eta_{R}$ are some values at a reference point (in laboratory conditions).

Using condition (64) and (43), we find that the transition from laminar to turbulent convection occurs at

$$
\nu_{R, \mathrm{tr}} \sim \frac{l_{0}^{2} \dot{e}_{R}}{R e_{\mathrm{cr}}}\left(\frac{\alpha g F}{\rho c_{p} l_{0}^{2} \dot{e}_{R}^{3}}\right)^{(2-\beta) / 3} .
$$

For $\beta=1$ we get the criterion for the constant viscosity convection.

\section{Bingham Rheology}

In this case the rheological law is approximated by the equation

$$
\tau_{i j}=\tau_{y}+\eta_{\infty} \dot{e}_{i j}
$$

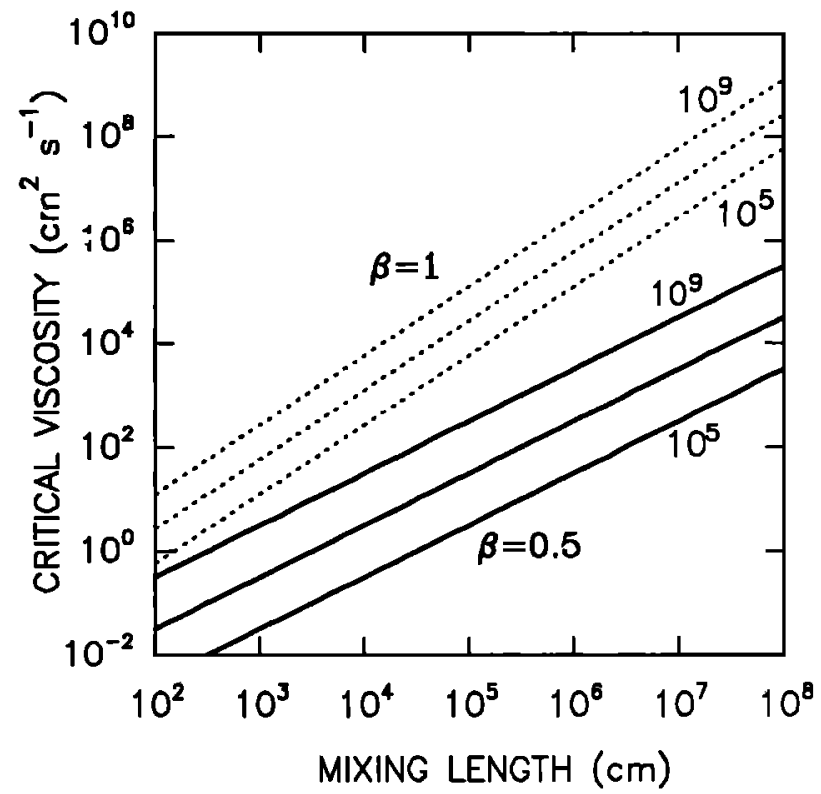

Fig. 7. Transition from turbulent to laminar convection for constant and power law viscosity with $\beta=0.5\left(\tau \sim \dot{e}^{\beta}\right)$ in terms of a critical viscosity measured in laboratory conditions, at a reference shear rate $10^{2} \mathrm{~s}^{-1}$. The dependence on the mixing length is shown.

where $\tau_{y}$ is the yield stress and $\eta_{\infty}$ is a constant, or in a more general case, it can be a function of the shear rate.

Consider only the case $\tau_{y} \gg \eta_{\infty} \dot{e}_{i j}$, when the rheology is essentially different from the Newtonian one (in the opposite case the rheology is effectively a Newtonian one with the viscosity $\left.\eta_{\infty}\right)$. The transition is determined by the criterion

$$
\frac{\rho u_{0}^{2}}{\tau_{y}} \sim R e_{\mathrm{cr}}
$$

or

$$
\tau_{y, t r} \sim \frac{\rho}{R e_{\mathrm{er}}}\left(\frac{\alpha g l_{0} F}{\rho c_{p}}\right)^{2 / 3}
$$

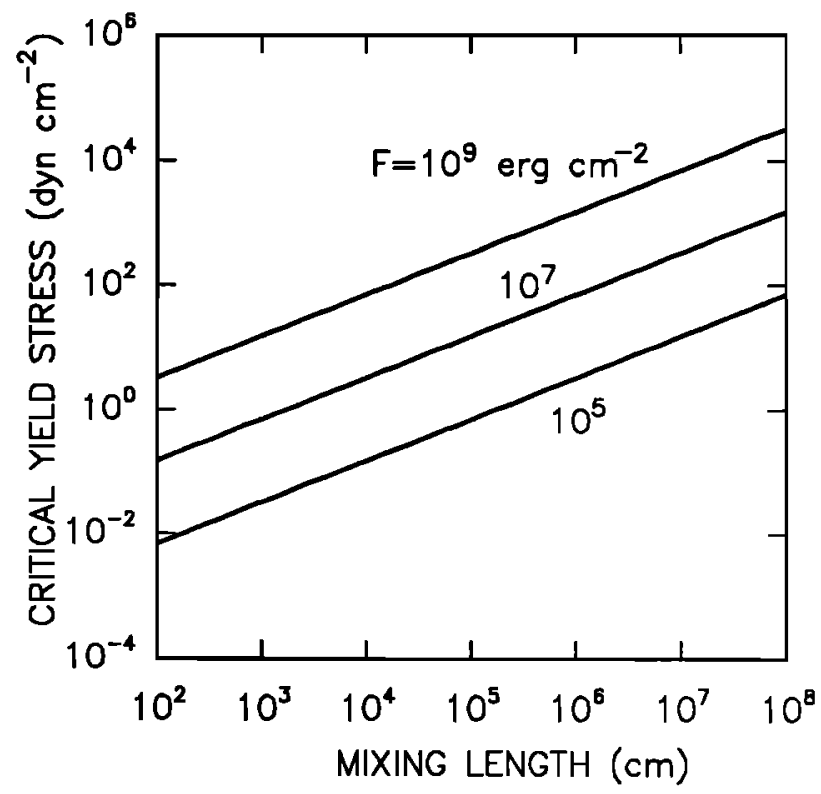

Fig. 8. Transition from turbulent to laminar convection for a Bingham rheology in terms of the critical yield stress. 


\section{Estimates for a Magma Ocean}

Figures 7 and 8 show the boundaries for the transition from laminar to turbulent convection depending on the mixing length for the rheologies considered above. The mixing length can be limited by rotation at $10^{5} \mathrm{~cm}$. If the rheology is assumed to be Newtonian, then the critical values of the viscosity (which in this case is independent of shear rate) follow the curves with $\beta=1$ (Figure 7 ). This is a reference value of the viscosity which is measured in laboratory conditions (at a reference strain rate of $10^{2} \mathrm{~s}^{-1}$ ) and extrapolated to high pressures. In the case of power law viscosity with some typical value $\beta=0.5$, the critical viscosity at $\dot{e}_{R}=10^{2} \mathrm{~s}^{-1}$ is by $2-5$ orders of magnitude less than the reference viscosity (Figure 7). This is the result of an increase of the effective (shear-rate dependent) viscosity when we scale from the relatively high shear rates of laboratory conditions to the much lower shear rates of the magma ocean. If $l_{0} \sim 10^{5} \mathrm{~cm}$ then turbulence does not occur if the reference viscosity is larger than $\sim 10^{2} \mathrm{P}$. This is a possible situation.

If a yield stress does exist (for this problem it means that the viscosity beyond the yield stress is so high that the convection is certainly laminar) it is estimated to be about $10^{3}-10^{4}$ dyn $\mathrm{cm}^{-2}$ [Robson, 1967; Shaw et al., 1968; Shaw, 1969; Pinkerton and Sparks, 1978; Ryerson et al., 1988]. This value can be more than the critical one for the possible values of the mixing length (Figure 8 ) and the turbulence ceases already at $20-30 \%$ of the crystal fraction, i.e., the minimum crystal fraction at which the non-Newtonian behavior is observed. The consequence for the suspension problem is that suspension above this crystal fraction could be impossible. Future results on the rheology of partial melts at very slow deformation rates would help to clarify this problem.

\section{Density Stratification in a Magma Ocean due to the Crystal Settling and the Suspension Problem}

A density stratification of a magma ocean unavoidably occurs when the crystals are present. This is due to a tendency for the crystals to sediment (up or down) even in the case of well-developed turbulence. An important feature of the density stratification due to sedimentation of the particles is that the layer cannot be rehomogenized as in the case of a layer stratified with the help of a chemical component.

The effect can be estimated from the balance between the sedimentation mass flux and the mass flux due to the turbulent diffusion. If the net particle flux from the system is zero, then there is a stationary regime described approximately as [e.g., Marsh and Maxey, 1985]

$$
u_{p} N \approx D_{t} \frac{\partial N}{\partial z}
$$

where $u_{p}$ is the relative velocity between the crystals and the melt, $N$ is the concentration of the particles, $D_{t}=u_{0} l_{0}$ is a characteristic turbulent diffusion for the convective flow with the mixing length $l_{0}$ [e.g., Landau and Lifshitz, 1989] and the corresponding velocity $u_{0}$, and $z$ is the vertical coordinate in the direction opposite to the sedimentation flux. Consider the case $u_{p} \ll u_{0}, \phi \ll 1$, where $\phi$ is the mass crystal fraction, and $\Delta \rho / \rho \ll 1$, where $\Delta \rho$ is the density difference between the liquid and the solid and assume for simplicity that the crystal distribution is a nearly constant one in an equilibrium. The particle concentration and mean density gradients are

$$
\begin{gathered}
\frac{\partial N}{\partial z} \approx-\frac{u_{p} N}{u_{0} l_{0}}, \\
\frac{1}{\rho} \frac{\partial \rho}{\partial z} \approx-\phi \frac{\Delta \rho}{\rho} \frac{u_{p}}{u_{0}} \frac{1}{l_{0}} .
\end{gathered}
$$

In the presence of a density gradient, $l_{0}$ must be determined by the characteristic distance at which the positive buoyancy of a fluid element $\alpha \delta T$ due to the temperature fluctuations is equal to the negative buoyancy due to the composition (the net buoyancy is zero):

$$
|\alpha \delta T| \sim\left|\frac{l_{0}}{\rho} \frac{\partial \rho}{\partial z}\right| .
$$

Substituting ( 43) and ( 53) (for arbitrary $l_{0}$ ), we find that the criterion for these two buoyancies to be equal to each other is independent of $l_{0}$ :

$$
\frac{\alpha F}{c_{p} u_{p} \phi \Delta \rho} \sim 1 \text {. }
$$

This means that the collapse of all turbulent scales down to the smallest (Kolmogorov) scale occurs at the same time. The above criterion coincides with the definition of the Froude number and it can be written as

$$
F r=\frac{u_{0}}{d \omega} \sim 1
$$

where

$$
\omega=\left(\frac{g}{\rho} \frac{\partial \rho}{\partial z}\right)^{1 / 2}
$$

is the Brunt-Väisälä frequency. It is a well-known criterion for the turbulence collapse in stratified fluids (see review by Hopfinger [1987]). Thus we arrive at the following conclusion: the turbulence is essentially the same as without any stratification when $F r \gg 1$ and there is no turbulence when $F r \ll 1$.

An important result is that (77) coincides with the energetic criterion for suspension (10) (ignoring numerical factors): the turbulence ceases due to the crystal settling at the same time as the suspension does. The conclusion is independent of the mixing length and thus it is possibly not influenced by the effects considered in previous sections.

\section{CONCLUSION}

1. If there is an effective mechanism of reentrainment from the bottom of a convective layer, the suspension is controlled by energetics of convection. Depending on the ratio $E_{p} / E$ between the energy dissipation due to the crystal settling and the total power supply to the layer (e.g., the total power produced by cooling of a magma ocean), three regimes can be distinguished:

Absolute sedimentation,

$$
E_{p} / E>1,
$$

Partial sedimentation,

$$
\epsilon D<E_{p} / E<1,
$$

Absolute suspension,

$$
E_{p} / E<\epsilon D
$$


where $D=\alpha g d / c_{p}$ is the dissipation parameter of the convective layer and $\epsilon<1$ is an efficiency factor which is probably about $1 \%$. This value is uncertain and is estimated from an experiment by Martin and Nokes [1988] and from the experiments on chemical erosion in convective layers [Olson, 1984]. In the absence of local mechanisms of re-entrainment, the third regime can not exist and it is identical with the second one. It can be formally treated as $\epsilon=0$.

2. The basic features of these regimes are the following. In the case of a steady heating of simple suspensions (no phase changes), the rates of settling in the first two regimes are similar. Variations can be in the mode of settling, depending on some other criteria (e.g., criterion of Koyaguchi et al. [1990]). However, in the case of cooling of partially molten systems, the first regime implies a complete differentiation because the dissipational heating due to the settling would exceed the heat loss from the system and increase of the crystal fraction must necessarily be compensated by settling. The total crystal fraction in the magma ocean is then small and is controlled mostly by the balance between the dissipation and the heat loss. The cooling can proceed only together with a continuous differentiation and a drop in the liquidus curve. The second regime in the case of cooling of partially molten systems is characterized by competition between the rate of crystallization, the rate of sedimentation, and the rate of turbulent diffusion, which determines the degree of differentiation. This is the regime studied by $A b e$ [1991, 1992]. The third regime implies an indefinitely longlived suspension. In the case of partially molten systems, the differentiation begins only near the maximum packing crystal fraction.

3. The dependence of the criteria separating regimes on the crystal fraction has a minimum near $\phi \sim 10 \%$ crystal fraction and is weak in comparison with uncertainties in the crystal radius, viscosity, and the heat flux. This minimum is due to decrease of dissipation at low $\phi$ and due to decrease of the settling velocity at high $\phi$. Together with the fact that the crystal fraction varies slowly with depth, this allows us to ignore these variations and to suggest some estimates in terms of crystal radius. The absolute sedimentation takes place at $r>10^{-2}-1 \mathrm{~cm}$ for the crystallization of deep layers and at $10^{-3}-10^{-1} \mathrm{~cm}$ for the crystallization of shallow layers (when the heat flux becomes smaller). The absolute suspension can take place at $r<10^{-3}-10^{-1} \mathrm{~cm}$ and at $r<10^{-4}-10^{-2} \mathrm{~cm}$ correspondingly if $\epsilon \sim 1 \%$. The region between these ranges is occupied by partial sedimentation and differentiation. Note that for deep magma oceans $D \sim 1$, which reduces the range occupied by partial differentiation relative to laboratory situations where $D \ll 1$.

4. The remaining problem is which local mechanism is responsible for the reentrainment in convective layers. We consider turbulence as such a mechanism [Tonks and Melosh, 1990] (noting, however, that the one re-entrainment case in the experiments by Martin and Nokes [1988] was quite far from the turbulent regime and the reentrainment was accomplished by some other mechanism). A simple model of turbulence predicts that the turbulence in the magma ocean ceases at $10^{9}-10^{10} \mathrm{P}$. This provides suspension until the maximum packing crystal fraction is reached (if the energetic criterion is satisfied). Rotation only reduces these values to $10^{5}-10^{8} \mathrm{P}$. Non-Newtonian rheology of partial melts can exclude turbulent convection already at $20-30 \%$ of the crystal fraction. After the cessation of turbulence, the evolution might involve partial sedimentation unless other mechanisms of reentrainment are involved.
5. In addition, it is found that the criterion for an absolute suspension coincides with the criterion requiring that the density stratification due to the crystal settling does not stop the turbulent convection.

Acknowledgments. The authors thank anonymous reviewers for their comments. This work was supported by the National Science Foundation grant EAR-89-16611.

\section{REFERENCES}

Abe, Y., Thermal evolution and chemical differentiation of the terrestrial magma ocean, paper presented at the IUGG Symposium on Chemical Evolution of the Earth and Planets, Int. Union Geod. and Geophys., Vienna, 1991.

Abe, Y., Thermal evolution and chemical differentiation of the terrestrial magma ocean, Workshop on the Physics and Chernistry of Magma Oceans from 1 bar to 4 Mbar, LPI Tech. Rep. 92-03, edited by C. B. Agee and J. Longhi, Pp. 9-10, Lunar and Planetary Inst., Houston, Texas, 1992.

Abe, Y., and T. Matsui, Early evolution of the Earth: Accretion, atmosphere formation, and thermal history, J. Geophys. Res., 91, E291-E302, 1986.

Andrade, E. N. C., Viscosity of liquids, Proc. Roy. Soc. London, $A, 215,36-43,1952$.

Bartlett, R. B., Magma convection, temperature distribution, and differentiation, Am. J. Sci., 267, 1067-1082, 1969.

Benz, W., and A. G. W. Cameron, Terrestrial effects of the giant impact, in Origin of the Earth, pp. 61-67, edited by N. E. Newsom and J. H. Jones, Oxford University Press, New York, 1990.

Benz, W., W. L. Slattery, and A. G. W. Cameron, The origin of the Moon and the single impact hypothesis, I, Icarus, 66, 515-535, 1986.

Benz, W., W. L. Slattery, and A. G. W. Cameron, The origin of the Moon and the single impact hypothesis, II, Icarus, 71, 30-45, 1987.

Benz, W., A. G. W. Cameron, and H. J. Melosh, The origin of the Moon and the single impact hypothesis, III, Icarus, 81, 113-131, 1989.

Bird, R. B., W. E. Stewart, and E. N. Lighfoot, Transport Phenomena, John Wiley, New York, 1960.

Bottinga, Y. and D. F. Weill, The viscosity of magmatic silicate liquids: A model for calculation, Am. J. Sci., 272, 438-475, 1972.

Boubnov, B. M., and G. S. Golitsyn, Experimental study of convective structures in rotating fluids, J. Fluid Mech., 167, 503$531,1986$.

Boubnov, B. M., and G. S. Golitsyn, Temperature and velocity field regimes of convective motions in a rotating plane fluid layer, J. Fluid Mech., 219, 215-239, 1990.

Busse, F. H., Nonlinear properties of thermal convection, Rep. Prog. Phys., 41, 1929-1967, 1978.

Cabot, W., V. M. Canuto, O. Hubickyj, and J. B. Pollack, The role of turbulent convection in the primitive solar nebula, I, Theory, Icarus, 69, 387-422, 1987.

Cameron, A. G. W., and W. Benz, Possible scenarios resulting from the giant impact, Lunar Planet. Sci. Conf. 20, 137-138, 1989.

Cameron, A. G. W., and W. Benz, The origin of the Moon and the single impact hypothesis IV, Icarus, 92, 204-216, 1991.

Castaing, B., G. Gunaratne, F. Heslot, L. Kadanoff, A. Libchaber, S. Thomae, X.-Z. Wu, S. Zaleski, and G. Zanetti, Scaling of hard thermal turbulence in Rayleigh-Benard convection, $J$. Fluid Mech., 204, 1-30, 1989.

Chandrasekhar, S., The stability of a layer of fluid heated below and subject to Coriolis forces, Proc. R. Soc. London Ser. A, $217,306-327,1953$.

Chandrasekhar, S., Hydrodynamic and Hydromagnetic Stability, Oxford, at the Clarendon Press, London, 1961.

Chen, R., H. J. S. Fernando, and D. L. Boyer, Formation of isolated vortices in a rotating convecting fluid, J. Geophys. Res., $94,18,445-18,453,1989$.

Clayton, D. D., Principles of Stellar Evolution and Nucleosynthesis, McGraw-Hill, New York, 1968.

Dikarev, S. N., On the influence of rotation on the convective 
structure in a deep homogeneous fluid, Dokl. Akad. Nauk SSSR, 273, 718-720, 1983.

Fernando, H. J. S., R. R. Chen, and D. L. Boyer, Effects of rotation on convective turbulence, J. Fluid Mech., 228, 513-547, 1991.

Flasar, F. M., and F. Birch, Energetics of core formation: A correction, J. Geophys. Res., 78, 6101-6103, 1973.

Gans, R. F., Viscosity of the Earth's core, J. Geophys. Res., 77, 360-366, 1972.

Garon, A. M., and R. J. Goldstein, Velocity and heat transport measurements of thermal convection, Phys. Fluids, 16, 1818$1825,1973$.

Golitsyn, G. S., Simple theoretical and experimental study of convection with some geophysical applications and analogies, J. Fluid Mech., 95, 567-608, 1978.

Golitsyn, G.S., Geostrophic convection, Dokl. Akad. Nauk SSSR, $251,1356-1360,1980$.

Golitsyn, G. S., Structure of convection in rapid rotation, Dokl. Akad. Nauk SSSR, 261, 317-320, 1981.

Golitsyn, G. S., Convection regimes on various rotating geophysical and astrophysical objects, Izv. Akad. Nauk SSSR, 27, 13$21,1991$.

Heslot, F., B. Castaing, and A. Libchaber, Transition to turbulence in helium gas, Phys. Rev. A, 36, 5870-5873, 1987.

Hewitt, J. M., D. P. McKenzie, and N. O. Weiss, Dissipative heating in convective flows, J. Fluid Mech., 68, 721-738, 1975. Hopfinger, E. J., Turbulence in stratified fluids: A review, $J$. Geophys. Res., 92, 5287-5303, 1987.

Hopfinger, E. J., Turbulence and vortices in rotating fluids, in Theoretical and Applied Mechanics, edited by P. Germain, M. Piau, and D. Caillerie, pp. 117-138, Elsevier, New York, 1989.

Hopfinger, E. J., F. K. Browand, and Y. Gagne, Turbulence and waves in a rotating tank, J. Fluid Mech., 125, 505-534, 1982.

Huppert, H. E., and R. S. J. Sparks, The fluid dynamics of a basaltic magma chamber replenished by influx of hot, dense ultrabasic magma, Contrib. Minerol. Petrol., 75, 279-289, 1980.

Kasting, J. F., Runaway and moist greenhouse atmosphere and the evolution of Earth and Venus, Icarus, 74, 472-494, 1988.

Kato, T., A. E. Ringwood, and T. Irifune, Experimental determination of element partioning between silicate perovskites, garnets and liquids: Constraints on early differentiation of the mantle, Earth Planet. Sci. Lett., 89, 123-145, 1988 a.

Kato, T., A. E. Ringwood, and T. Irifune, Constraints on element partition coefficients between $\mathrm{MgSiO}_{3}$ perovskite and liquid determined by direct measurements, Earth Planet. Sci. Lett., $90,65-68,1988 b$.

Koyaguchi, T., M. A. Hallworth, H. E. Huppert, and R. S. J. Sparks, Sedimentation of particles from a convecting fluid, Nature, 343, 447-450, 1990.

Kraichnan, R. H., Turbulent thermal convection at arbitrary Prandtl number, Phys. Fluids, 5, 1374-1389, 1962.

Kushiro, I., Viscosity, density, and structure of silicate melts at high pressures, and their petrological applications, in Physics of Magmatic Processes, edited by R. B. Hargraves, pp. 93-120, Princeton University Press, Princeton, N. J., 1980.

Kushiro, I., Viscosity of partial melts in the upper mantle. $J$. Geophys. Res., 91, 9343-9350, 1986.

Lambeck, K., The Earth's Variable Rotation, Cambridge University Press, New York, 1980.

Landau, L. D., and E. M. Lifshitz, Fluid Mechanics, Pergamon, New York, 1989.

Marsh, B. D., and M. R. Maxey, On the distribution and separation of crystals in convecting magma, $J$. Volcanol. Geotherm. Res., 24, 95-150, 1985.

Martin, D., and R. Nokes, Crystal settling in a vigorously convecting magma chamber, Nature, 392, 534-536, 1988.

Martin, D., and R. Nokes, A fluid dynamical study of crystal settling in convecting magmas, J. Petrol., 30, 1471-1500, 1989.

Matsui, T., and Y. Abe, Formation of a "magma ocean" on the terrestrial planets due to the blanketing effect of an impactinduced atmosphere, Earth Moon Planets, 34, 223-230, 1986.

McBirney, A. R., and T. Murase, Rheological properties of magmas, Annu. Rev. Earth Planet. Sci., 12, 337-357, 1984.

McKenzie, D., The generation and compaction of partially molten rock, J. Petrol., 25, 713-765, 1984.

Melosh, H. J., Giant impacts and the thermal state of the early Earth, in Origin of the Earth, edited by N. E. Newsom and J. H. Jones, pp. 69-83, Oxford University Press, New York, 1990.
Miller, G. H., E. M. Stolper, and T. J. Ahrens, The equation of state of a molten komatiite, 1 , Shock wave compression to 36 GPa, J. Geophys. Res., 96, 11,831-11,848, 1991 a.

Miller, G. H., E. M. Stolper, and T. J. Ahrens, The equation of state of a molten komatiite, 2, Application to komatiite petrogenesis and the hadean mantle, J. Geophys. Res., 96, 11,849-11,864, $1991 b$.

Nakagawa, Y., and P. Frenzen, A theoretical and experimental study of cellular convection in rotating fluids, Tellus, 7, 1-21, 1955 .

Newsom, H. E., and S. R. Taylor, Geochemical implications of the formation of the Moon by a single great impact, Nature, 338, 29-34, 1989.

Olson, P., An experimental approach to thermal convection in a two-phase mantle, J. Geophys. Res., 89, 11,293-11,301, 1984.

Persikov, E. S., V. A. Zharikov, P. G. Bukhtiyarov, and S. F. Polskoy, The effect of volatiles on the properties of magmatic melts, Eur. J. Mineral., 2, 621-642, 1990.

Pinkerton, H., and R. S. J. Sparks, Field measurement of the rheology of lava, Nature, 276, 383-385, 1978.

Priestly, C. H. B., Turbulent Transfer in the Lower Atmosphere, University of Chicago Press, Chicago, Ill., 1959.

Ringwood, A. E., Earliest history of the Earth-Moon system, in Origin of the Earth, edited by N. E. Newsom and J. H. Jones, pp. 101-134, Oxford University Press, New York, 1990.

Robson, G. R., Thickness of Etnean lavas, Nature, 216, 251-252, 1967.

Rossby, H. T., A sudy of Benard convection with and without rotation, J. Fluid Mech., 36, 309-335, 1969.

Rudman, M., Two-phase natural convection: Implications for crystal settling in magma chambers, Phys. Earth Planet. Inter., 72, 153-172, 1992 .

Ryan, N. W., and M. M. Johnson, Transition from laminar to turbulent tlow in pipes, A.I. Ch. E. Journal, 5, 433-435, 1959.

Ryerson, F. J., H. C. Weed, and A. J. Piwinskii, Rheology of subliquidus magmas, I, Picritic compositions, J. Geophys. Res., 93, 3421-3436, 1988.

Safronov, V. S., The heating of the Earth during its formation, Icarus, 39, 3-12, 1978.

Shaw, H. R., Rheology of basalt in the melting range, J. Petrol., $10,510-535,1969$.

Shaw, H. R., Viscosity of magmatic silicate liquids: An empirical method of prediction, Am. J. Sci., 266, 255-264, 1972.

Shaw, H. R., T. L. Wright, D. L. Peck, and R. Okamura, The viscosity of basaltic magma: An analysis of field measurements in Makaopuhi lava lake, Hawaii, Am. J. Sci., 266, 225-264, 1968.

Sleep, N. H., Gradual entrainment of a chemical layer at the base of the mantle by overlaying convection, Geophys. J., 95, 437$447,1988$.

Solomatov, V. S., Parameterization of temperature- and stressdependent viscosity convection and the thermal evolution of Venus, in Flow and Dynamic Modeling of the Earth and Planets, University of Alaska, Fairbanks, in press, 1993.

Solomatov, V. S., and D. J. Stevenson, Models of magma rheology, Eos Trans. AGU, 72, suppl., 573-574, 1991.

Solomatov, V. S., and D. J. Stevenson, Non fractional crystallization of a terrestrial magma ocean, J. Geophys. Res., this volume $(a)$.

Solomatov, V. S., and D. J. Stevenson, Kinetics of crystal growth in a terrestrial magma ocean, $J$. Geophys. Res., this volume (b).

Soo, S. H., Fluid Dynamics of Multiphase Systems, Blaisdell, Waltham, Mass., 1967.

Stevenson, D. J., Origin of the Moon - The collision hypothesis, Annu. Rev. Earth Planet. Sci., 15, 271-315, 1987.

Taylor, G. J., and M. D. Norman, Evidence for magma oceans on asteroids, the Moon, and Earth, Workshop on the Physics and Chemistry of Magma Oceans from 1 bar to $4 \mathrm{Mbar}, L P I$ Tech. Rep. 92-03, edited by C. B. Agee and J. Longhi, pp. 58-65, Lunar and Planetary Inst., Houston, Texas, 1992.

Thompson, C., and D. J. Stevenson, Gravitational instabilities in 2-phase disks and the origin of the Moon, Astrophys. J., 333, 452-481, 1988.

Tonks, W. B., and H. J. Melosh, The physics of crystal settling and suspension in a turbulent magma ocean, in Origin of the Earth, edited by N. E. Newsom and J. H. Jones, Pp. 151-174, Oxford University Press, New York, 1990.

Turcotte, D. L., and G. Schubert, Geodynamics: Applications of 
Continuum Physics to Geological Problems, John Wiley, New York, 1982.

Verhoogen, J., Energetics of the Earth, National Academy of Sciences, Washington, D. C., 1980.

Weinstein, S. A., D. A. Yuen, and P. L. Olson, Evolution of crystal-settling in magma-chamber convection, Earth Planet. Sci. Lett., 87, 237-248, 1988.

Wetherill, G. W., Ocurrence of giant impacts during the growth of the terrestrial planets, Science, 228, 877-879, 1985.

Wetherill, G. W., Formation of the Earth, Annu. Rev. Earth Planet. Sci., 18, 205-256, 1990.
Zahnle, K. J., J. F. Kasting, and J. B. Pollack, Evolution of a steam atmosphere during Earth's accretion, Icarus, 74, 62-97, 1988.

V. S. Solomatov and D. J. Stevenson, Division of Geological and Planetary Sciences, California Institute of Technology, Pasadena, CA 91125.

(Received May 5, 1992;

revised December 9, 1992 ,

accepted December 15, 1992.) 